\title{
GENERALIZED LEFSCHETZ NUMBERS
}

\author{
BY
}

\author{
S. Y. HUSSEINI
}

\begin{abstract}
Given $[C ; f]$, where $C$ is a finitely-generated $\pi$-projective chain complex, and $f: C \rightarrow C$ a $(\pi, \varphi)$-chain map, with $\varphi: \pi \rightarrow \pi$ being a homomorphism, then the generalized Lefschetz number $L_{(\pi, \varphi)}[C ; f]$ of $[C ; f]$ is defined as the alternating sum of the $(\pi, \varphi)$-Reidemeister trace of $f$. In analogy with the ordinary Lefschetz number, $L_{(\pi, \varphi)}[C ; f]$ is shown to satisfy the commutative property and to be invariant under $(\pi, \varphi)$-chain homotopy. Also, when $H_{*} C$ is $\pi$-projective,

$$
L_{(\pi, \varphi)}[C ; f]=L_{(\pi, \varphi)}\left[H_{*} C ; H_{*} f\right] .
$$

If $\pi^{\prime} \subset \pi$ is $\varphi$-invariant and with finite index, then for $\alpha \in \pi^{\prime}$, the $\left(\pi^{\prime}, \varphi\right)$ Reidemeister class $\left[\alpha ; \pi^{\prime}\right]$ is essential for $f: C \rightarrow C$ if and only if $[\alpha ; \pi]_{\varphi}$ is essential. If $\pi^{\prime} \subset \pi$ is normal, then one can use the cosets of $\pi \bmod \pi^{\prime}$ to detect the essential $(\pi, \varphi)$-classes of $f: C \rightarrow C$. This is expressed as a decomposition of $L_{(\pi, \varphi)}[C ; f]$ in terms of $L_{\left(\pi^{\prime}, \varphi_{\xi}\right)}\left[C^{\prime} ; f_{\xi}\right]$ where $f(\cdot) \xi^{-1}=f_{\xi}(\cdot)$ and $\varphi_{\xi}(\cdot)=\xi \varphi(\cdot) \xi^{-1}$. The algebraic theory is applied to the Nielsen theory of a map $f: X \rightarrow X$, where $X$ is a finite $\mathrm{CW}$-complex relative to a regular cover $\tilde{X}^{\prime \prime} \rightarrow X$. One can define a generalized Lefschetz number $L_{\left(\pi^{\prime \prime}, \varphi^{\prime \prime}\right)}$ using any cellular approximation to $f$, where $\pi^{\prime \prime}$ is the group of covering transformations of $\tilde{X}^{\prime \prime} \rightarrow X$. The quantity $L_{\left(\pi^{\prime \prime}, \varphi^{\prime \prime}\right)}$ can be expressed naturally as a formal sum in the $\pi^{\prime \prime}$-Nielsen classes of $f$ with their indices appearing as coefficients. From this expression, one is able to deduce from the properties of the generalized Lefschetz number the usual results of the relative Nielsen theory.
\end{abstract}

Introduction. Suppose that $C$ is a finitely-generated $\mathbf{K}[\pi]$-projective graded module, where $\pi$ is a group and $\mathbf{K}$ a commutative ring with a unity. If $f: C \rightarrow C$ is a K-homomorphism such that $f(x \sigma)=f(x) \varphi(\sigma)$ for all $x$ in $C$ and $\sigma$ in $\pi$, we shall say that $f$ is a $(\pi, \varphi)$-map. The generalized Lefschetz number $L_{(\pi, \varphi)}[C ; f]$ of $[C ; f]$ is defined to be the alternating sum of the Reidemeister trace of $f$ [7]. The quantity $L_{(\pi, \varphi)}[C ; f]$ lies in $\mathbf{K} R_{\varphi}[\pi]$, the free $\mathbf{K}$-module generated by the $(\pi, \varphi)$-Reidemeister classes of $\pi$. The aim of this article is to study algebraically the quantity $L_{(\pi, \varphi)}[C ; f]$ : Does it satisfy the commutative property? Is it chain-homotopy invariant when $C$ is a chain complex? Can it be computed at the homology level? How is $L_{(\pi, \varphi)}[C ; f]$ related to $L_{\left(\pi^{\prime}, \varphi\right)}[C, f]$ when $\pi^{\prime}$ is a $\varphi$-invariant subgroup of $\pi$ ?

These quantities arise naturally in the fixed point theory of nonsimply connected finite polyhedra. Suppose that $f: X \rightarrow X$ is a map of the finite polyhedron $X$ to itself. If $f$ is simplicial, then using any lift of $f$ to the universal cover one obtains at the

Received by the editors February 29, 1980 and, in revised form, April 1, 1981. Presented at the 775th meeting of the American Mathematical Society, Fixed Point Theory: Special Session, Bloomington, Indiana, April 11-12, 1980.

1980 Mathematics Subject Classification. Primary 55M20, 55U99; Secondary 20 J05. 
chain level a $(\pi, \varphi)$-map with $\pi$ being the group of covering transformations. In [8] Wecken defined a formal sum $R_{0}(f)$ in the essential Nielsen classes of $f$, with the coefficients being the usual indices of these classes, and noted that if $f$ is replaced by a simplicial map $f^{s}$ with isolated fixed points and whose fixed simplices are maximal, then the formal sum $R_{0}(f)$ can be interpreted as the generalized Lefschetz number of $f^{s}$. But the questions of whether $R_{0}(f)$ can be computed in terms of any simplicial approximation, or at the homology level, were left open. We shall see that the answer to these questions are positive to the first without any restriction and to the second when the homology of the covering space is projective over $\pi$. In [3], an obstruction $o(f) \in H^{m}(X ; \Re)$, where $\mathscr{B}$ is a certain local coefficient system, to deforming $f$ into a fixed point free map was defined and evaluated on the equivariant fundamental class to yield $R_{0}(f)$. It was also shown that if $\varphi: \pi \rightarrow \pi$ has finite image, then $R_{0}(f)$ can be described as a trace-like quantity $\varrho_{\pi}(f)$ in terms of the subgroups $\pi_{\alpha} \pi^{\prime} / \pi^{\prime}$ acting on the homology groups of the cover corresponding to $\pi^{\prime}$, where $\pi^{\prime}=\operatorname{ker} \varphi$, and $\pi_{\alpha}=\left\{\sigma \in \pi \mid \sigma \alpha \varphi\left(\sigma^{-1}\right)=\alpha\right\}$. We shall see that $\mathfrak{L}_{\pi}(f)$ is actually a generalized Lefschetz number $L_{(\pi, \varphi)}$ and that $\varphi$ need not have a finite image. The results of [3] also suggest that there are nice and natural relations between $L_{(\pi, \varphi)}$ and that of its subgroups. This article is largely motivated by the search for such relations.

The main results are stated in $\$ 1$. The first set of results is concerned with the basic properties of $L_{(\pi, \varphi)}[C ; f]$. In analogy with the ordinary Lefschetz number it satisfies the commutativity property (Proposition (1.2)), is chain homotopy invariant (Proposition (1.3)), and if $H_{*} C$ is projective over $\mathbf{K}[\pi]$, then the Hopf Trace Theorem is valid (Proposition (1.4)).

The next set of results is concerned with the inductive properties of $L_{(\pi, \varphi)}$. If $\pi^{\prime}$ is $\varphi$-invariant and has finite index, then (Theorem 1.5) for $\alpha$ in $\pi^{\prime}$, a class $[\alpha ; \pi]_{\varphi}$ appears with a nontrivial coefficient in $L_{(\pi, \varphi)}[C ; f]$ if and only if it appears with a nontrivial coefficient in $L_{\left(\pi^{\prime}, \varphi\right)}[C, f]$. Moreover the coefficient in $L_{\left(\pi^{\prime}, \varphi\right)}$ is a multiple of that in $L_{(\pi, \varphi)}$ and the multiplicity can be described group-theoretically. Thus $L_{(\pi, \varphi)}[C, f]$ can be computed in terms of appropriate subgroups. Also Theorem (1.5) can be used in situations where it is convenient to vary the subgroup $\pi^{\prime}$ and the homomorphisms $\varphi$, for example, such as in the construction of the quantity $\mathcal{L}_{\pi}(f)$ (Definitions (6.13) and (6.28) of [3]). Further study of the inductive properties requires a more general trace-theory, one which reflects the module structure of $C$ over $\mathbf{K}\left[\pi^{\prime}\right]$. The object $[C ; f]$ is relativized accordingly: one specifies in $C$ a $\pi^{\prime}$-submodule $C^{\prime}$ which generates $C$ as a $\pi$-module. The values of the generalized Lefschetz number $L_{\left(\pi^{\prime}, \varphi\right)}\left[C, C^{\prime} ; f\right]$ is in $\mathbf{K} R_{\varphi}\left[\pi, \pi^{\prime}\right]$, where $R_{\varphi}\left[\pi, \pi^{\prime}\right]$ is the set of equivalence classes of $\pi$ by the action $\alpha \rightarrow \sigma \alpha \varphi(\sigma)^{-1}$ for $\sigma$ in $\pi^{\prime}$. If $\pi^{\prime}$ is normal, then $L_{\left(\pi^{\prime}, \varphi\right)}\left[C, C^{\prime} ; f\right]$ can be expressed as a sum $\Sigma_{\xi} L_{\left(\pi^{\prime}, \varphi_{\xi}\right)}\left[C^{\prime} ; f_{\xi}^{\prime}\right] \otimes \xi$ where $\xi$ ranges over a set of representatives of $\pi \bmod \pi^{\prime}, \varphi(\cdot)=\xi \varphi(\cdot) \xi^{-1}$ and $f_{\xi}^{\prime}$ is, roughly speaking, the restriction of $f_{\xi}(\cdot)=f(\cdot) \xi^{-1}$ to $C^{\prime} \subset C$. If moreover $\left[\pi: \pi^{\prime}\right]<\infty$, then one can deduce therefrom an expression for $\left|\pi^{\prime \prime}\right| L_{(\pi, \varphi)}[C ; f]$, where $\pi^{\prime \prime}=\pi / \pi^{\prime \prime}$, in terms of $L_{\left(\pi^{\prime}, \varphi_{\xi}\right)}[C ; f]$, the generalized Lefschetz numbers of $C$ instead of those of $C^{\prime}$. This generalizes Theorem (6.22) of [3]. 
The third set of results relates to the topological applications. A generalized Lefschetz number is more conveniently assigned to a commutative diagram

$$
\begin{array}{cccc} 
& \tilde{X}^{\prime \prime} & \stackrel{\tilde{f}^{\prime \prime}}{\rightarrow} & \tilde{X}^{\prime \prime} \\
S_{\left(\pi^{\prime \prime}, \varphi^{\prime \prime}\right)}[X, f]: & p^{\prime \prime} \downarrow & & \downarrow p^{\prime \prime} \\
& X & \stackrel{f}{\rightarrow} & X
\end{array}
$$

where $p^{\prime \prime}: \tilde{X}^{\prime \prime} \rightarrow X$ is a regular cover, $\pi^{\prime \prime}$ its group of covering transformations, and $\varphi^{\prime \prime}: \pi^{\prime \prime} \rightarrow \pi^{\prime \prime}$ is the homomorphism such that $\tilde{f}^{\prime \prime}(\tilde{x} \sigma)=\tilde{f}^{\prime \prime}(\tilde{x}) \varphi^{\prime \prime}(\sigma)$ for all $\tilde{x}$ in $\tilde{X}^{\prime \prime}$ and $\sigma$ in $\pi^{\prime \prime}$. Here $X$ is assumed to be a finite CW-complex, and $\tilde{X}^{\prime \prime}$ to have the induced cellular structure. If one approximates $f$ by a cellular map $f^{c}$, then the lift of $f^{c}$ provides a $\left(\pi^{\prime \prime}, \varphi^{\prime \prime}\right)$-chain map $C\left(\tilde{f}^{c}\right)^{\prime \prime}: C_{*} \tilde{X}^{\prime \prime} \rightarrow C_{*} \tilde{X}^{\prime \prime}$ of the cellular chain groups. It is shown that the generalized Lefschetz number $L_{\left(\pi^{\prime \prime}, \varphi^{\prime \prime}\right)}[X, f]=$ $L_{\left(\pi^{\prime \prime}, \varphi^{\prime \prime}\right)}\left[C_{*} \tilde{X}^{\prime \prime} ; C_{*}\left(\tilde{f}^{c}\right)^{\prime \prime}\right]$ is well defined, commutative (Proposition (1.12)), homotopy invariant (Proposition (1.11)). It is also independent of the cellular structure on $X$. Theorem (1.13) asserts that if $L_{\left(\pi^{\prime \prime}, \varphi^{\prime \prime}\right)} S[X, f] \neq 0$, then $f: X \rightarrow X$ has essential fixed points, and, moreover if $X$ is also a Wecken complex, and $\tilde{X}^{\prime \prime} \rightarrow X$ is the universal cover, then $L_{\left(\pi^{\prime \prime}, \varphi^{\prime \prime}\right)} S[X, f]=0$ implies that $f$ is homotopic to a fixed-point free map. Theorem (1.13) generalizes Theorem (6.26) of [3] because $\varphi: \pi \rightarrow \pi$ is arbitrary, and for the first assertion, $X$ is a finite CW-complex and not just a polyhedron.

In $\$ 2$ we give examples where $L_{(\pi, \varphi)} \neq 0$ but the ordinary Lefschetz number $L=0$. The examples are given to illustrate the methods of the paper. The second of these examples is really the same as McCord's [5], except that it is presented in a different manner. Other examples could have been easily constructed, but it seemed of interest to show the relative ease of computation afforded by the methods of this paper.

In the remaining sections, the proofs of the results stated in $\S 1$ are given. I would like to thank Ed Fadell for many useful and stimulating conversations pertaining to this paper.

1. Statement of results. Suppose that $\pi$ is a group and let $\varphi: \pi \rightarrow \pi$ be a homomorphism. Then a subgroup $\pi^{\prime}$ of $\pi$ acts on $\pi$ on the right according to the rule

$$
\alpha \mapsto \sigma^{-1} \alpha \varphi(\sigma)
$$

where $\sigma \in \pi^{\prime}$ and $\alpha \in \pi$. Note that $\varphi$ is not required to take $\pi^{\prime}$ to itself. This action is, by definition, the $\left(\pi^{\prime}, \varphi\right)$-Reidemeister action of $\pi^{\prime}$ on $\pi$. Denote the $\left(\pi^{\prime}, \varphi\right)$-orbits by $R_{\varphi}\left[\pi, \pi^{\prime}\right]$, and when $\pi=\pi^{\prime}$, by $R_{\varphi}[\pi]$. Let $\mathbf{K}$ be a commutative ring with unity, and denote by $\mathrm{K} R_{\varphi}[\pi]$ and $\mathrm{K} R_{\varphi}\left[\pi, \pi^{\prime}\right]$ the free $\mathrm{K}$-module generated by $R_{\varphi}[\pi]$ and $R_{\varphi}\left[\pi, \pi^{\prime}\right]$ respectively.

Now let $\mathfrak{R}_{p \times q}(\pi)$ be the set of $(p \times q)$-matrices with entries in $\mathbf{K}[\pi]$, the group-ring of $\pi$ over $\mathbf{K}$. Observe that if $\beta=\sigma^{-1} \alpha \varphi(\sigma)$, where $\sigma \in \pi$, then $\alpha=\zeta \xi$ and $\beta=\xi \varphi(\zeta)$, where $\xi=\sigma^{-1} \alpha$ and $\zeta=\sigma$. Hence the natural map

$$
\mathbf{K}[\pi] \rightarrow \mathbf{K} R_{\varphi}\left[\pi, \pi^{\prime}\right]
$$


which takes $\alpha$ to $\left[\alpha ; \pi, \pi^{\prime}\right]_{\varphi}$, its $\left(\pi^{\prime}, \varphi\right)$-orbit in $R_{\varphi}\left[\pi, \pi^{\prime}\right]$, has the property that

$$
\left[\zeta \xi ; \pi, \pi^{\prime}\right]_{\varphi}=\left[\xi \varphi(\zeta) ; \pi, \pi^{\prime}\right]_{\varphi}
$$

for all $\xi \in \pi$ and $\zeta \in \pi^{\prime}$. This fact leads easily to a trace-like function.

Proposition (1.1). There is a unique function

$$
\operatorname{tr}_{\left(\pi^{\prime}, \varphi\right)}: \Re_{p, p}(\pi) \rightarrow \mathbf{K} R_{\varphi}\left[\pi, \pi^{\prime}\right]
$$

such that

$$
\operatorname{tr}_{\left(\pi^{\prime}, \varphi\right)}(M+N)=\operatorname{tr}_{\left(\pi^{\prime}, \varphi\right)}(M)+\operatorname{tr}_{\left(\pi^{\prime}, \varphi\right)}(N)
$$

for all $M, N$ in $\Re_{p, p}(\pi)$;

$$
\operatorname{tr}_{\left(\pi^{\prime}, \varphi\right)}(M N)=\operatorname{tr}_{\left(\pi^{\prime}, \varphi\right)}\left(N M^{\varphi}\right)
$$

for all $M$ in $\mathfrak{R}_{q, p}(\pi)$ and $N$ in $\Re_{p, q}\left(\pi^{\prime}\right)$, where $M^{\varphi}$ is obtained from $M$ by applying $\varphi$ to each of the entries of $M$; and

$$
\operatorname{tr}_{\left(\pi^{\prime}, \varphi\right)}(M)=\sum_{i}\left[m_{i i} ; \pi, \pi^{\prime}\right]_{\varphi}
$$

where $\left[m_{i i} ; \pi, \pi^{\prime}\right]_{\varphi}$ is the class of $m_{i i}$ in $\mathbf{K} R_{\varphi}\left[\pi, \pi^{\prime}\right]$.

Note that Condition 3 defines $\operatorname{tr}_{\left(\pi^{\prime}, \varphi\right)}(M)$ uniquely. The rest of the proof is straightforward and is left for the reader (cf. [6]).

A finitely-generated $\pi^{\prime}$-based right $\mathbf{K}[\pi]$-projective module is, by definition, a pair $\left(P, P^{\prime}\right)$ such that

(i) $P$ is a right $\mathrm{K}[\pi]$-module;

(ii) $P^{\prime}$ is a finitely-generated right $\mathrm{K}\left[\pi^{\prime}\right]$-projective submodule of $P$; and

(iii) the natural imbedding $P^{\prime} \rightarrow P$ induces an isomorphism

$$
P^{\prime} \otimes_{\mathbf{K}\left[\pi^{\prime}\right]} \mathbf{K}[\pi] \rightarrow P
$$

of $\mathbf{K}[\pi]$-modules where $\mathbf{K}[\pi]$ acts naturally on the right on $P^{\prime} \otimes_{\mathbf{K}\left[\pi^{\prime}\right]} \mathbf{K}[\pi]$. Note that this implies that $P$ is itself a projective finitely-generated $\mathbf{K}[\pi]$-module.

If $P$ is a finitely-generated free $\mathbf{K}[\pi]$-module, then $P$ can be turned into a $\pi^{\prime}$-based module by choosing a $\pi$-base $\{x\}$ and then defining $P^{\prime}$ to be the $\pi^{\prime}$-submodule of $P$ generated by $\{x\}$. A K-homomorphism

$$
f: P \rightarrow \cdot P
$$

is said to be a $(\pi, \varphi)$-homomorphism if and only if

$$
f(x \boldsymbol{\sigma})=f(x) \varphi(\sigma)
$$

for all $x \in P$ and $\sigma \in \pi$. Now if $P^{\prime}$ is free over $\pi^{\prime}$ and if $\left\{x_{1}, \ldots, x_{p}\right\}$ is a $\pi^{\prime}$-basis for $P^{\prime}$, then $f$ defines in the usual way a matrix $A$ with entries in $\mathbf{K}[\pi]$. By definition, let

$$
\operatorname{tr}_{\left(\pi^{\prime}, \varphi\right)}\left[P, P^{\prime} ; f\right]=\operatorname{tr}_{\left(\pi^{\prime}, \varphi\right)}(A) .
$$

It is easy to show, as in [6], that the element $\operatorname{tr}_{\left(\pi^{\prime}, \varphi\right)}\left[P, P^{\prime} ; f\right]$ of $\mathbf{K} R_{\varphi}\left[\pi, \pi^{\prime}\right]$ is independent of the choice of the $\pi^{\prime}$-basis of $P^{\prime}$. It will be shown in $\S 3$ that $\operatorname{tr}_{\left(\pi^{\prime}, \varphi\right)}\left[P, P^{\prime} ; f\right]$ can be also defined when $P^{\prime}$ is projective but not necessarily free. 
The preceding concepts can be naturally extended to graded modules. A finitelygenerated $\mathbf{K}\left[\pi, \pi^{\prime} ; \varphi\right]$-projective graded right module is a triple $\left[C, C^{\prime} ; f\right]$ where

(i) $C$ is a graded right $\mathbf{K}[\pi]$-module;

(ii) $C^{\prime}$ is a graded right $\mathbf{K}\left[\pi^{\prime}\right]$-submodule $\sum_{i=0}^{n} C_{i}^{\prime}$ of $C$ such that, for each $i,\left[C_{i}, C_{i}^{\prime}\right]$ is a finitely-generated $\pi^{\prime}$-based $\mathbf{K}[\pi]$-projective right module; and

(iii) $f: C \rightarrow C$ is a $(\pi, \varphi)$-map of degree 0 .

If $\left[C, C^{\prime} ; f\right]$ is a finitely-generated $\mathbf{K}\left[\pi, \pi^{\prime} ; \varphi\right]$-projective graded right module then, by definition, let

$$
L_{\left(\pi^{\prime}, \varphi\right)}\left[C, C^{\prime} ; f\right]=\sum_{i=0}^{n}(-1)^{i} \operatorname{tr}_{\left(\pi^{\prime}, \varphi\right)}\left[C_{i}, C_{i}^{\prime} ; f_{i}\right]
$$

be the generalized Lefschetz number of $\left[C, C^{\prime} ; f\right]$. Note that $f$ is not required to take $C^{\prime}$ to itself. If $\pi^{\prime}=\pi$, then $C=C^{\prime}$ in which case we write $\left[C^{\prime} ; f\right]$ for $\left[C, C^{\prime} ; f\right]$.

If $C$ is a $\mathbf{K}[\pi]$-chain complex, and $f$ a chain map, then we shall call $[C ; f]$ a $\mathbf{K}[\pi, \varphi]$-chain complex. Two $\mathbf{K}[\pi]$-chain maps $f: C \rightarrow C$ and $g: C \rightarrow C$ are said to be $(\pi, \varphi)$-chain homotopic if and only if there is a $(\pi, \varphi)$-map $\delta: C \rightarrow C$ such that

$$
\delta \partial+\partial \delta=f-g .
$$

The following proposition describes the commutativity property of $L_{\left(\pi, \pi^{\prime} ; \varphi\right)}$. To formulate this property, suppose that $\varphi_{1}: \pi_{1} \rightarrow \pi_{2}$ and $\varphi_{2}: \pi_{2} \rightarrow \pi_{1}$ are homomorphisms, and let $\pi_{1}^{\prime} \subset \pi_{1}$ and $\pi_{2}^{\prime} \subset \pi_{2}$ be subgroups. Assume that $\varphi_{1}\left(\pi_{1}^{\prime}\right) \subset \pi_{2}^{\prime}$. Then the correspondence $\sigma \mapsto \varphi_{1}(\sigma)$ induces a homomorphism

$$
\left(\varphi_{1}\right)_{*}: \mathbf{K} R_{\varphi_{2} \varphi_{1}}\left[\pi_{1}, \pi_{1}^{\prime}\right] \rightarrow \mathbf{K} R_{\varphi_{1} \varphi_{2}}\left[\pi_{2}, \pi_{2}^{\prime}\right]
$$

Proposition (1.2). Suppose that $\left(C_{1}, C_{1}^{\prime}\right)$ and $\left(C_{2}, C_{2}^{\prime}\right)$ are finitely-generated $\mathbf{K}\left[\pi_{1}, \pi_{1}^{\prime}\right]-$ and $\mathbf{K}\left[\pi_{2}, \pi_{2}^{\prime}\right]$-projective graded right modules, and let $f: C_{1} \rightarrow C_{2}$ and $g$ : $C_{2} \rightarrow C_{1}$ be $\left(\pi_{1}, \varphi_{1}\right)$ - and $\left(\pi_{2}, \varphi_{2}\right)$-maps respectively. If $f\left(C_{1}^{\prime}\right) \subset C_{2}^{\prime}$, then

$$
\left(\varphi_{1}\right)_{*}\left(L_{\left(\pi_{1}, \pi_{1}^{\prime} ; \varphi_{2} \varphi_{1}\right)}\left[C_{1}, C_{1}^{\prime} ; g f\right]=L_{\left(\pi_{2}, \pi_{2}^{\prime} ; \varphi_{1} \varphi_{2}\right)}\left[C_{2}, C_{2}^{\prime} ; f g\right]\right.
$$

Proposition (1.2) implies the chain-homotopy invariance of $L_{(\pi, \varphi)}[C, f]$.

Proposition (1.3). Suppose that $[C ; f]$ and $[C ; g]$ are two finitely-generated $\mathbf{K}[\pi, \varphi]$-projective complexes, and assume that $f: C \rightarrow C$ and $g: C \rightarrow C$ are $(\pi, \varphi)$-chain homotopic. Then

$$
L_{(\pi, \varphi)}[C ; f]=L_{(\pi, \varphi)}[C ; g]
$$

The proofs of Propositions (1.2) and (1.3) are given in §3. We shall also show there how to deduce the following version of the Hopf Trace Theorem from Propositions (1.2) and (1.3).

Proposition (1.4). Suppose that $[C ; f]$ is a finitely-generated $\mathbf{K}[\pi, \varphi]$-projective chain complex, and assume that $H_{*} C$ is $\mathbf{K}[\pi]$-projective. Then

$$
L_{(\pi, \varphi)}[C ; f]=L_{(\pi, \varphi)}\left[H_{*} C, H_{*} f\right] .
$$


Note that Proposition (1.4) applies, for example, when $\mathbf{K}$ has characteristic zero and $\pi$ is finite.

Now let

$$
q: \mathbf{K} R_{\varphi}\left[\pi, \pi^{\prime}\right] \rightarrow \mathbf{K} R_{\varphi}[\pi]
$$

be the homomorphism induced by sending $\left[\alpha ; \pi, \pi^{\prime}\right]_{\varphi}$ to $[\alpha ; \pi]_{\varphi}$. If $\left[C, C^{\prime} ; f\right]$ is a $\mathbf{K}\left[\pi, \pi^{\prime} ; \varphi\right]$-graded module, then it is easy to see that

$$
q\left(L_{\left(\pi, \pi^{\prime} ; \varphi\right)}\left[C, C^{\prime} ; f\right]\right)=L_{(\pi, \varphi)}[C ; f]
$$

where $[C ; f]$ is the complex obtained by disregarding $C^{\prime}$.

Before taking up the problem of how $L_{(\pi, \varphi)}$ is related to $L_{\left(\pi^{\prime}, \varphi\right)}$, let us observe that in computing $L_{(\pi, \varphi)}[C ; f]$ we can divide out the kernel of $\varphi$. For if we denote $\operatorname{ker} \varphi$ by $\pi^{\prime}$ we can easily see that the natural projection $p: \pi \rightarrow \pi^{\prime \prime}=\pi / \pi^{\prime \prime}$ induces an isomorphism $p_{*}: \mathbf{K} R_{\varphi}[\pi] \rightarrow \mathbf{K} R_{\varphi^{\prime \prime}}\left[\pi^{\prime \prime}\right]$, where $\varphi^{\prime \prime}: \pi^{\prime \prime} \rightarrow \pi^{\prime \prime}$ is the homomorphism induced by $\varphi$. Moreover, a simple calculation shows that $p_{*}$ takes $L_{(\pi, \varphi)}[C ; f]$ to $L_{\left(\pi^{\prime \prime}, \varphi^{\prime \prime}\right)}[\bar{C} ; \bar{f}]$ where $\bar{C}=C \otimes_{\mathbf{K}\left[\pi^{\prime}\right]} \mathbf{K}$ and $\bar{f}=f \otimes_{\mathbf{K}\left[\pi^{\prime}\right]}$ l. In particular if $\left|\pi^{\prime \prime}\right|<\infty$ we can compute in terms of $\left[H_{*} \bar{C}, H_{*} \bar{f}\right]$ as a consequence of Proposition (1.4).

We wish to describe how $L_{(\pi, \varphi)}[C ; f]$ is related to $L_{\left(\pi^{\prime}, \varphi\right)}[C ; f]$. If $\alpha \in \pi$, denote by $\pi_{\alpha}$ the stability subgroup $\left\{\sigma \in \pi \mid \sigma \alpha \varphi(\sigma)^{-1}=\alpha\right\}$.

Theorem (1.5). Suppose that $\varphi\left(\pi^{\prime}\right) \subset \pi^{\prime}$ and that $\left[\pi: \pi^{\prime}\right]<\infty$. Assume that $[C ; f]$ is a finitely-generated $\mathbf{K}[\pi, \varphi]$-projective graded module, and let $\alpha \in \pi^{\prime}$. Then

$$
\lambda_{\left[\alpha ; \pi^{\prime}\right]_{\varphi}}=\mu_{\left[\alpha ; \pi^{\prime}\right]_{\varphi}} \lambda_{[\alpha ; \pi]_{\varphi}}
$$

where $\lambda_{\left[\alpha ; \pi^{\prime}\right]_{\varphi}}$ is the coefficient of $\left[\alpha ; \pi^{\prime}\right]_{\varphi}$ in $L_{\left(\pi^{\prime}, \varphi\right)}[C ; f], \lambda_{[\alpha ; \pi]_{\varphi}}$ is the coefficient of $[\alpha ; \pi]_{\varphi}$ in $L_{(\pi, \varphi)}[C ; f]$, and $\mu_{\left[\alpha ; \pi^{\prime}\right]_{\varphi}}=\left[\pi_{\alpha}: \pi_{\alpha}^{\prime}\right]$ with $\pi_{\alpha}^{\prime}=\pi_{\alpha} \cap \pi^{\prime}$.

The proof will be given in $\S 4$.

Roughly speaking, Theorem (1.5) is a generalization of the well-known result about the Euler characteristic of a finite cover. Actually if $f$ is the identity map of $C$, and $C$ is free over $\mathbf{K}[\pi]$, then the theorem is just that, since $[1 ; \pi]_{\varphi}$ is the only class with a nontrivial coefficient in the generalized Lefschetz number, and the coefficient is the Euler characteristic. Also Theorem (1.5) allows us to extend certain properties of $\pi^{\prime}$ to finite extensions. As an illustration, consider the following extension of Gottlieb's Theorem $[4,6]$. First let us say that a $(\pi, \varphi)$-Reidemeister class $[\alpha ; \pi]_{\varphi}$ is $(\pi, \varphi)$-essential for $[C ; f]$ if and only if the coefficient of $[\alpha ; \pi]_{\varphi}$ in $L_{(\pi, \varphi)}[C ; f]$ is nontrivial.

COROllaRY (1.6). Suppose that the $(\pi, \varphi)$-complex $[C ; f]$ is a finitely-generated $\mathbf{Z}[\pi ; \varphi]$-complex such that $H_{*} C=\mathbf{Z}$, where $\mathbf{Z}$ denotes the integers, and let $[\alpha, \pi]_{\varphi}$ be a $(\pi, \varphi)$-essential class for $[C ; f]$. If $\left[\pi\right.$ : Fix $\left.\varphi_{\alpha}\right]<\infty$, then

$$
\text { Fix } \varphi_{\alpha} \cap \operatorname{cent}\left(\operatorname{im} \varphi_{\alpha}\right)=\{1\}
$$

where Fix $\varphi_{\alpha}=\left\{\sigma \in \pi \mid \varphi_{\alpha}(\sigma)=\sigma\right\}$, and cent $\left(\operatorname{im} \varphi_{\alpha}\right)$ is the centralizer of $\operatorname{im} \varphi_{\alpha}$ in $\pi$.

For the proof see $\$ 4$.

Gottlieb's Theorem is the special case when $C$ is free, $\varphi: \pi \rightarrow \pi$ is the identity, and $f$ induces the identity $H_{*} C \cong \mathbf{Z} \rightarrow H_{*} C \cong \mathbf{Z}$. For then one can show that $f$ is 
$(\pi, \varphi)$-chain homotopic to the identity map $C \rightarrow C$ [see Lemma (3.4) of §3]. Hence $L_{(\pi, \varphi)}[C ; f] \neq 0$ is just the condition that the Euler characteristic of $C$ is $\neq 0$, and $\alpha=1$.

Thus, if the characteristic of $\mathbf{K}$ is zero, an immediate corollary of Theorem (1.5) is that, for $\alpha \in \pi^{\prime}$, the class $[\alpha ; \pi]_{\varphi}$ is $(\pi, \varphi)$-essential if and only if $\left[\alpha ; \pi^{\prime}\right]_{\varphi}$ is $\left(\pi^{\prime}, \varphi\right)$-essential. Hence the $(\pi, \varphi)$-essential classes of $f$ and their coefficients can be obtained by means of the $\varphi$-invariant subgroups $\pi^{\prime}$ of $\pi$, whose index is finite. Since there is only a finite number of such classes it is sufficient in general to compute $L_{(\pi, \varphi)}$ in terms of a finite number of $\varphi$-invariant subgroups of finite index. For example, if $|\pi|<\infty, \varphi$ is the identity, and the ground ring $\mathbf{K}$ is of characteristic zero, then $L_{(\pi, \varphi)}$ is determined by $L_{\left(\pi^{\prime}, \varphi\right)}$ when $\pi^{\prime}$ ranges over the cyclic subgroups of $\pi$.

If the subgroup $\pi^{\prime}$ in Theorem (1.5) is a subgroup of Fix $\varphi$, then the theorem allows one to reduce the computation, for those classes with representatives in $\pi^{\prime}$, to the more standard situation when $f: C \rightarrow C$ is $\pi$-equivariant. Sometimes it is possible to account for every class $[\alpha ; \pi]_{\varphi}$ by reducing the problem to the $\pi$-equivariant case, if one is willing to vary $\varphi$ and $f: C \rightarrow C$. In fact, the correspondence $\gamma \mapsto \gamma \alpha^{-1}$ defines an isomorphism $\alpha_{*}: \mathbf{K} R_{\varphi}[\pi] \rightarrow \mathbf{K} R_{\varphi_{\alpha}}[\pi]$, where $\varphi_{\alpha}(\cdot)=\alpha \varphi(\cdot) \alpha^{-1}$. Also $L_{(\pi, \varphi)}[C ; f]$ goes to $L_{\left(\pi, \varphi_{\alpha}\right)}\left[C ; f_{\alpha}\right]$ where $f_{\alpha}(\cdot)=f(\cdot) \alpha^{-1}$, with $[\alpha, \pi]_{\varphi}$ going to $[1, \pi]_{\varphi_{\alpha}}$. It is easy to see that

$$
\operatorname{Fix}\left(\varphi_{\alpha}\right)=\pi_{\alpha}
$$

and that $\varphi_{\alpha}\left(\pi_{\alpha}\right) \subset \pi_{\alpha}$. Now if $\mid$ image $\varphi \mid<\infty$, we see that the subgroups $(\operatorname{ker} \varphi) \pi_{\alpha} \subset \pi$ are all with finite index and for each $\alpha, \varphi_{\alpha}\left((\operatorname{ker} \varphi) \pi_{\alpha}\right) \subset(\operatorname{ker} \varphi) \pi_{\alpha}$. Thus, $(\operatorname{ker} \varphi) \pi_{\alpha}$ detects $\left[1 ;(\operatorname{ker} \varphi) \pi_{\alpha}\right]_{\varphi_{\alpha}}$ according to Theorem (1.5) and hence, $[\alpha, \pi]_{\varphi}$. This is how $\varrho_{\pi}(f)$ of [3] was constructed.

If $\pi^{\prime}$ is normal in $\pi$ and $\varphi\left(\pi^{\prime}\right) \subset \pi^{\prime}$, then one can use the cosets of $\pi^{\prime}$ to detect the essential classes of a $(\pi, \varphi)$-map $f: C \rightarrow C$ as follows. First choose a set $Z=\{\zeta\}$ of coset representatives of $\pi \bmod \pi^{\prime}$, and define

$$
Z_{*}: \mathbf{K} R_{\varphi}\left[\pi, \pi^{\prime}\right] \rightarrow \sum_{\zeta} \mathbf{K} R_{\varphi_{\zeta}}\left[\pi^{\prime}\right] \otimes \zeta
$$

to be the homomorphism induced by sending $\left[\gamma ; \pi, \pi^{\prime}\right]_{\varphi}$ to $\left[\gamma \zeta^{-1} ; \pi^{\prime}\right]_{\varphi_{\zeta}} \otimes \zeta$, where $\varphi_{\zeta}(\cdot)=\zeta \varphi(\cdot) \zeta^{-1}$. If $\left[C, C^{\prime} ; f\right]$ is a finitely-generated $\mathbf{K}\left[\pi, \pi^{\prime} ; \varphi\right]$-projective graded module, then we have by definition the isomorphism

$$
C \cong \sum_{\zeta} C^{\prime} \otimes_{\mathbf{K}\left[\pi^{\prime}\right]} \mathbf{K}\left[\pi^{\prime} \zeta\right]
$$

induced by the imbedding $C^{\prime} \stackrel{i}{\rightarrow} C$, where $\zeta$ ranges over $Z$. Let $j: C \rightarrow C^{\prime}=C^{\prime}$ $\otimes_{\mathbf{K}\left[\pi^{\prime}\right]} \mathbf{K}\left[\pi^{\prime}\right]$ be the $\mathbf{K}\left[\pi^{\prime}\right]$-projection defined by the preceding decomposition, and define

$$
f_{\zeta}^{\prime}: C^{\prime} \rightarrow C^{\prime}
$$

to be the composite $C^{\prime} \stackrel{i}{\rightarrow} C \stackrel{f_{\zeta}}{\rightarrow} C \stackrel{j}{\rightarrow} C^{\prime}$, where $f_{\zeta}(\cdots)=f(\cdots) \zeta^{-1}$. Then $\left[C^{\prime} ; f_{\zeta}^{\prime}\right]$ is a finitely-generated $\mathbf{K}\left[\pi^{\prime} ; \varphi_{\zeta}\right]$-projective graded module. 
THEOREM (1.7). With the notations and assumptions given above, we have

$$
Z_{*} L_{\left(\pi^{\prime}, \varphi\right)}\left[C, C^{\prime} ; f\right]=\sum_{\zeta} L_{\left(\pi^{\prime}, \varphi_{\zeta}\right)}\left[C^{\prime} ; f_{\zeta}^{\prime}\right] \otimes \zeta
$$

where $\zeta$ ranges over $Z$.

The proof is given in $\$ 5$. A consequence of the theorem is the following result. Suppose that $\Xi=\{\xi\} \subset \pi$ is a set of coset representatives of $\pi \bmod \pi^{\prime}$, and define

$$
\Xi_{*}^{\prime}: \sum_{\xi} \mathrm{K} R_{\varphi_{\xi}}\left[\pi^{\prime}\right] \otimes \xi \rightarrow \mathrm{K} R_{\varphi}[\pi]
$$

to be the homomorphism induced by sending $\gamma$ to $\gamma \xi$.

TheOREM (1.8). Suppose that $\varphi:\left(\pi, \pi^{\prime}\right) \rightarrow\left(\pi, \pi^{\prime}\right)$ is a homomorphism and assume that $\pi^{\prime}$ is normal in $\pi$ and with finite index $\left[\pi: \pi^{\prime}\right]$. Let $[C ; f]$ be a finitely-generated $\mathbf{K}[\pi, \varphi]$-projective graded module. Then the homomorphism $\Xi_{*}^{\prime}$ defined above takes $L_{\left(\pi^{\prime}, \varphi_{\xi}\right)}\left[C ; f_{\xi}\right] \otimes \xi$, where $f_{\xi}(\cdot)=f(\cdot) \xi^{-1}$, to a linear combination in those $(\pi, \varphi)$-classes of $f: C \rightarrow C$ which meet the coset $\pi^{\prime} \xi$ nontrivially, and

$$
\left|\pi^{\prime \prime}\right| L_{(\pi, \varphi)}[C ; f]=\Xi_{*}^{\prime}\left(\sum_{\xi} L_{\left(\pi^{\prime}, \varphi_{\xi}\right)}\left[C ; f_{\xi}\right] \otimes \xi\right)
$$

where $\pi^{\prime \prime}=\pi / \pi^{\prime}$.

The proof is given in $\$ 5$.

The first corollary is of the Jiang-type: it describes $L_{(\pi, \varphi)}$ in terms of a single $L_{\left(\pi^{\prime}, \varphi\right)}$ where $\pi^{\prime}$ is a normal subgroup of $\pi$.

Corollary (1.9). Suppose that for all $\xi \in \Xi, \varphi_{\xi}\left|\pi^{\prime}=\varphi\right| \pi^{\prime}$. Then

$$
\left|\pi^{\prime \prime}\right| L_{(\pi, \varphi)}[C ; f]=\Xi_{*}^{\prime}\left(\sum_{\xi} L_{\left(\pi^{\prime}, \varphi\right)}\left[C ; f_{\xi}\right] \otimes \xi\right) .
$$

Moreover, if $[C ; f]$ is a complex and the maps $f_{\xi}: C \rightarrow C$ are $\left(\pi^{\prime}, \varphi\right)$-chain homotopic, then

$$
\left|\pi^{\prime \prime}\right|\left(L_{(\pi, \varphi)}[C ; f]\right)=\Xi_{*}^{\prime} L_{\left(\pi^{\prime}, \varphi\right)}[C ; f]\left(\sum_{\xi} 1 \otimes \xi\right) .
$$

Hence, in this case, $L_{(\pi, \varphi)}[C ; f]=0$ if and only if $L_{\left(\pi^{\prime}, \varphi\right)}[C ; f]=0$.

The condition that $\varphi_{\xi}\left|\pi^{\prime}=\varphi\right| \pi^{\prime \prime}$ for all $\xi \in \Xi$ is satisfied, for example, when $\pi=\pi^{\prime} \times \pi^{\prime \prime}$ and $\Xi=\pi^{\prime \prime}$, or when $\pi^{\prime}$ is central. The other condition that the maps $f_{\xi}$ are $\left(\pi^{\prime \prime}, \varphi\right)$-chain homotopic is satisfied when, say, $H_{*} C \cong \mathbf{K}$ (cf. the proof of Proposition (3.4) of $\S 3$ ).

Next observe that the natural projection $p: \pi \rightarrow \pi^{\prime \prime}$ induces a homomorphism

$$
p_{*}: \mathbf{K} R_{\varphi}[\pi] \rightarrow \mathbf{K} R_{\varphi^{\prime \prime}}\left[\pi^{\prime \prime}\right] \text {. }
$$

It is easy to verify that if $[C ; f]$ is a projective $\mathbf{K}[\pi ; \varphi]$-chain complex, then $[\bar{C} ; \bar{f}]$ is a projective $\mathbf{K}\left[\pi^{\prime \prime} ; \varphi^{\prime \prime}\right]$-chain complex, where $\bar{C}=C \otimes_{\mathbf{K}\left[\pi^{\prime}\right]} \mathbf{K}$ and

$$
\bar{f}=f \otimes_{\mathbf{K}\left[\pi^{\prime}\right]} 1: \bar{C} \rightarrow \bar{C} .
$$


Also, the splitting of Theorem (1.8) is natural with respect to $p: \pi \rightarrow \pi^{\prime \prime}$, and consequently we obtain the following corollary.

Corollary (1.10). Let $C=C \otimes_{\mathbf{K}\left[\pi^{\prime}\right]} \mathbf{K}$ and $f=f \otimes_{\mathbf{K}\left[\pi^{\prime}\right]} 1$. Then

$$
\left|\pi^{\prime \prime}\right| L_{\left(\pi^{\prime \prime}, \varphi^{\prime \prime}\right)}[\bar{C} ; \bar{f}]=\sum_{\xi} L\left(\bar{f}_{\xi^{\prime \prime}}\right) \otimes \xi^{\prime \prime}
$$

where $\xi^{\prime \prime}$ ranges over $\pi^{\prime \prime}$ and $L\left(\bar{f}_{\xi^{\prime \prime}}\right)$ is the ordinary Lefschetz number of $\bar{f}_{\xi^{\prime \prime}}(\cdot)=$ $\bar{f}(\cdot) \xi^{\prime \prime-1}$.

Note that if $\pi$ is finite and one takes $\pi^{\prime \prime}=\{1\}$, then Corollary (1.10) is essentially Theorem (6.22) of [3].

Now let us consider the applications to fixed-point theory. Let $f: X \rightarrow X$ be a map of the finite CW-complex $X$ to itself, and let $p^{\prime \prime}: \tilde{X}^{\prime \prime} \rightarrow X$ be a regular cover. Assume that there is a lift $\tilde{f}^{\prime \prime}: \tilde{X}^{\prime \prime} \rightarrow \tilde{X}^{\prime \prime}$ of $f$. Then there is a unique homomorphism $\varphi$ : $\pi^{\prime \prime} \rightarrow \pi^{\prime \prime}$ where $\pi^{\prime \prime}$ is the group of covering transformations of $p^{\prime \prime}: X^{\prime \prime} \rightarrow X$, such that

$$
\tilde{f}^{\prime \prime}(x \boldsymbol{\sigma})=\tilde{f}^{\prime \prime}(x) \varphi(\sigma)
$$

for all $x \in X^{\prime \prime}$ and $\sigma \in \pi^{\prime \prime}$. We shall call such maps $\left(\pi^{\prime \prime}, \varphi^{\prime \prime}\right)$-maps, and denote the whole given data by the commutative diagram:

$$
\begin{array}{cccc} 
& \tilde{X}^{\prime \prime} & \stackrel{\tilde{f}^{\prime \prime}}{\rightarrow} & \tilde{X}^{\prime \prime} \\
S_{\left(\pi^{\prime \prime}, \varphi^{\prime \prime}\right)}[X, f]: & p^{\prime \prime} \downarrow & & \downarrow p^{\prime \prime} \\
& X & \stackrel{f}{\rightarrow} & X
\end{array}
$$

We shall call $S_{\left(\pi^{\prime \prime}, \varphi^{\prime \prime}\right)}[X, f]$ a $\left(\pi^{\prime \prime}, \varphi^{\prime \prime}\right)$-geometric setting of the map $f: X \rightarrow X$. Let us observe that if $\tilde{f}^{\prime \prime}$ is replaced by another lift $\tilde{f}_{\alpha}^{\prime \prime}(\cdots)=\tilde{f}^{\prime \prime}(\cdots) \alpha^{-1}$ then $\varphi^{\prime \prime}$ is replaced by $\varphi_{\alpha}^{\prime \prime}$ where $\varphi_{\alpha}^{\prime \prime}(\cdot)=\alpha \varphi^{\prime \prime}(\cdot) \alpha^{-1}$. Replace $f$ by a cellular approximation. Then there is a $\left(\pi^{\prime \prime}, \varphi^{\prime \prime}\right)$-homotopy of the setting $S_{\left(\pi^{\prime \prime}, \varphi^{\prime \prime}\right)}[X, f]$ to another where the lift is also cellular. Denote the cellular chain groups of $\tilde{X}^{\prime \prime}$ by $C_{*}\left(\tilde{X}^{\prime \prime}\right)$, and, by definition let the generalized Lefschetz number of the $\left(\pi^{\prime \prime}, \varphi^{\prime \prime}\right)$-setting $S_{\left(\pi^{\prime \prime}, \varphi^{\prime \prime}\right)}[X, f]$ be

$$
L_{\left(\pi^{\prime \prime}, \varphi^{\prime \prime}\right)} S[X, f]=L_{\left(\pi^{\prime \prime}, \varphi^{\prime \prime}\right)}\left[C_{*} \tilde{X}^{\prime \prime} ; C_{*} \tilde{f}^{\prime \prime}\right] .
$$

In $\$ 7$ we shall show that $L_{\left(\pi^{\prime \prime}, \varphi^{\prime \prime}\right)} S[X, f]$ is well defined and independent of the $\mathrm{CW}$-structure on $X$. Moreover it has the following two basic properties in analogy with the usual Lefschetz number.

Proposition (1.11) (Homotopy InVariance). If $S_{\left(\pi^{\prime \prime}, \varphi^{\prime \prime}\right)}[X, f]$ is $a\left(\pi^{\prime \prime}, \varphi^{\prime \prime}\right)$-setting, and $g: X \rightarrow X$ is homotopic to $f$, then there is a $\left(\pi^{\prime \prime}, \varphi^{\prime \prime}\right)$-setting $S_{\left(\pi^{\prime \prime}, \varphi^{\prime \prime}\right)}[X, g]$ homotopic to $S_{\left(\pi^{\prime \prime}, \varphi^{\prime \prime}\right)}[X, f]$ and

$$
L_{\left(\pi^{\prime \prime}, \varphi^{\prime \prime}\right)} S[X, f]=L_{\left(\pi^{\prime \prime}, \varphi^{\prime \prime}\right)} S[X, g]
$$

The other property is commutativity, appropriately formulated. Suppose therefore that $f: X \rightarrow Y$ and $g: Y \rightarrow Y$ are maps, where $X$ and $Y$ are finite $C W$-complexes, and 
let $\tilde{p}^{\prime \prime}: \tilde{X}^{\prime \prime} \rightarrow X$ and $q^{\prime \prime}: \tilde{Y}^{\prime \prime} \rightarrow Y$ be two finite CW-complexes. Assume that $\tilde{f}^{\prime \prime}$ : $\tilde{X}^{\prime \prime} \rightarrow \tilde{Y}^{\prime \prime}$ and $\tilde{g}^{\prime \prime}: \tilde{Y}^{\prime \prime} \rightarrow \tilde{X}^{\prime \prime}$ are lifts of $f$ and $g$ respectively. There are unique homomorphisms $\varphi^{\prime \prime}: \pi^{\prime \prime} \rightarrow \gamma^{\prime \prime}$ and $\psi^{\prime \prime}: \gamma^{\prime \prime} \rightarrow \pi^{\prime \prime}$, where $\pi^{\prime \prime}$ and $\gamma^{\prime \prime}$ are the groups of covering transformations of $p^{\prime \prime}: \tilde{X}^{\prime \prime} \rightarrow X$ and $q^{\prime \prime}: \tilde{Y}^{\prime \prime} \rightarrow Y$, respectively, such that $\tilde{f}^{\prime \prime}$ is a $\left(\pi^{\prime \prime}, \varphi^{\prime \prime}\right)$ - and $\tilde{g}^{\prime \prime}$ a $\left(\gamma^{\prime \prime}, \psi^{\prime \prime}\right)$-map. Thus we obtain the settings

$$
\begin{array}{cccc} 
& X^{\prime \prime} & \stackrel{\tilde{g}^{\prime \prime} \tilde{f}^{\prime \prime}}{\rightarrow} & X^{\prime \prime} \\
S_{\left(\pi^{\prime \prime}, \psi^{\prime \prime} \varphi^{\prime \prime}\right)}[X, g f]: & p \downarrow & & \downarrow p \\
& X & \stackrel{g f}{\rightarrow} & X
\end{array}
$$

and

$$
\begin{array}{cccc} 
& Y^{\prime \prime} & \stackrel{\tilde{f}^{\prime \prime} \tilde{g}^{\prime \prime}}{\rightarrow} & Y^{\prime \prime} \\
S_{\left(\gamma^{\prime \prime}, \varphi^{\prime \prime} \psi^{\prime \prime}\right)}[Y, f g]: & q \downarrow & & \downarrow q \\
& Y & \stackrel{f g}{\rightarrow} & Y
\end{array}
$$

Proposition (1.12) (The Commutative Property). Let

$$
\varphi_{*}^{\prime \prime}: \mathbf{K} R_{\psi^{\prime \prime} \varphi^{\prime \prime}}\left[\pi^{\prime \prime}\right] \rightarrow \mathbf{K} R_{\varphi^{\prime \prime} \psi^{\prime \prime}}\left[\gamma^{\prime \prime}\right]
$$

be the homomorphism induced by $\varphi^{\prime \prime}$. Then

$$
\varphi_{*}^{\prime \prime}\left(L_{\left(\pi^{\prime \prime}, \psi^{\prime \prime} \varphi^{\prime \prime}\right)} S[X, g f]\right)=L_{\left(\gamma^{\prime \prime}, \varphi^{\prime \prime} \psi^{\prime \prime}\right)} S[Y, f g] .
$$

For the proof of Propositions (1.12) and (1.13) see $\S 6$.

The geometric significance of $L_{\left(\pi^{\prime \prime}, \varphi^{\prime \prime}\right)}$ is summed up in the following generalization of Theorem (6.26) of [3], and is based on Wecken's special calculation [8].

THEOREM (1.13). Suppose that $S_{\left(\pi^{\prime \prime}, \varphi^{\prime \prime}\right)}[X, f]$ is a geometric $\left(\pi^{\prime \prime}, \varphi^{\prime \prime}\right)$-setting for the map $f: X \rightarrow X$, where $X$ is a finite $C W$-complex. Then

$$
L_{\left(\pi^{\prime \prime}, \varphi^{\prime \prime}\right)} S[X, f]=\sum \lambda_{[\alpha]}\left[\alpha ; \pi^{\prime \prime}\right]_{\varphi^{\prime \prime}}
$$

where $\lambda_{[\alpha]}$ is the usual index of the $\pi^{\prime \prime}$-Nielsen class $p^{\prime \prime}\left(\right.$ Fix $\left.\tilde{f}_{\alpha}^{\prime \prime}\right)$. Here $p^{\prime \prime}$ is the covering map $p^{\prime \prime}: \tilde{X}^{\prime \prime} \rightarrow X$ in the given setting and Fix $\tilde{f}_{\alpha}^{\prime \prime}$ is the fixed-point set of the lift $\tilde{f}_{\alpha}^{\prime \prime}(\cdots)=\tilde{f}_{\alpha}^{\prime \prime}(\cdots) \alpha^{-1}$.

Note that $\varphi^{\prime \prime}: \pi^{\prime \prime} \rightarrow \pi^{\prime \prime}$ is not required to have finite image. The proof of the theorem is not difficult; it amounts to a direct calculation of the generalized Lefschetz number $L_{\left(\pi^{\prime \prime}, \varphi^{\prime \prime}\right)} S[X, f]$ in the manner of [8] and will be indicated in $\S 6$. For the definition of $\pi^{\prime \prime}$-Nielsen classes see $\S 6$ of [3].

Theorem (1.13) implies, obviously, the Lefschetz theorem. Namely, if $L_{\left(\pi^{\prime \prime}, \varphi^{\prime \prime}\right)} S[X, f] \neq 0$ for some setting $S_{\left(\pi^{\prime \prime}, \varphi^{\prime \prime}\right)}[X, f]$ for the map $f: X \rightarrow X$, then $f$ has essential fixed points. Also Theorem (1.13) implies that if $X$ is a Wecken complex, then $L_{(\pi, \varphi)} S[X, f] \neq 0$ if and only if $f$ has no essential fixed points, where $S_{(\pi, \varphi)}[X, f]$ is a universal setting, in the sense that the cover of $X$ in $S_{(\pi, \varphi)}[X, f]$ is universal. 
One can conclude from Propositions (1.11) and (1.12) that the generalized Lefschetz numbers are homotopy-type invariants for maps $f: X \rightarrow X$ in the following sense.

Proposition (1.14). Suppose that $f: X \rightarrow X$ and $g: Y \rightarrow Y$ are maps of finite $C W$-complexes which are related by a homotopy-commutative diagram

$$
\begin{array}{lll}
X & \stackrel{f}{\rightarrow} & X \\
\downarrow h & & \downarrow h \\
Y & \stackrel{g}{\rightarrow} & Y
\end{array}
$$

where $h$ is a homotopy equivalence. Let $S_{\left(\gamma^{\prime \prime}, \psi^{\prime \prime}\right)}[Y, g]$ be a setting for $g: Y \rightarrow Y$. Then there is a setting $S_{\left(\pi^{\prime \prime}, \varphi^{\prime \prime}\right)}[X, f]$ for $f: X \rightarrow X$ such that

$$
h_{*} L_{\left(\pi^{\prime \prime}, \varphi^{\prime \prime}\right)} S[X, f]=L_{\left(\gamma^{\prime \prime}, \psi^{\prime \prime}\right)} S[Y, g]
$$

where $h_{*}: \mathbf{K} R_{\varphi^{\prime \prime}}\left[\pi^{\prime \prime}\right] \rightarrow \mathbf{K} R_{\psi^{\prime \prime}}\left[\gamma^{\prime \prime}\right]$ is an isomorphism induced by $h$.

The proof follows easily from the previous results, and is therefore left for the reader.

2. Examples where $L_{(\pi, \varphi)} \neq 0$ but $L=0$.

EXAmple (2.1). Let $\tilde{X}^{\prime \prime} \subset \mathbf{R}^{2}$ be the CW-complex which consists of the union of the vertical and horizontal unit segments with $\mathbf{R}^{2}$ being the Euclidean plane, and denote the group of translation $\mathbf{Z} \oplus \mathbf{Z}$ by $\pi^{\prime \prime}$. Then there is a $\left(\pi^{\prime \prime}, \varphi^{\prime \prime}\right)$-cellular map $\tilde{f}^{\prime \prime}: \tilde{X}^{\prime \prime} \rightarrow \tilde{X}^{\prime \prime}$ which leaves $(0,0)$ fixed, with $\varphi^{\prime \prime}: \pi^{\prime \prime} \rightarrow \pi^{\prime \prime}$ being the homomorphism which takes $\sigma$ to $\sigma^{3}$ and $\tau$ to $\tau^{-2}$. Here $\sigma$ and $\tau$ are, respectively, the horizontal and vertical translations. Then if we put $X=\tilde{X}^{\prime \prime} / \pi^{\prime \prime}$, we see that $\tilde{f}^{\prime \prime}$ induces a cellular map $f: X \rightarrow X$ which fits in the geometric $\left(\pi^{\prime \prime}, \varphi^{\prime \prime}\right)$-setting

$$
\begin{array}{cccc} 
& \tilde{X}^{\prime \prime} & \stackrel{\tilde{f}^{\prime \prime}}{\rightarrow} & \tilde{X}^{\prime \prime} \\
S_{\left(\pi^{\prime \prime}, \varphi^{\prime \prime}\right)}[X ; f]: & \downarrow p^{\prime \prime} & & \downarrow p^{\prime \prime} \\
& X & \stackrel{f}{\rightarrow} & X
\end{array}
$$

where $p^{\prime \prime}$ is the natural projection. An easy computation shows that

$$
R_{\varphi^{\prime \prime}}\left[\pi^{\prime \prime}\right] \cong \operatorname{coker}\left\{\varphi^{\prime \prime}-1: \pi^{\prime \prime} \rightarrow \pi^{\prime \prime}\right\} \cong\left\{\sigma \mid \sigma^{2}=1\right\} \times\left\{\tau \mid \tau^{3}=1\right\} .
$$

Put $\tilde{e}_{0}^{\prime \prime}=(0,0), \tilde{e}_{1}^{\prime \prime}=(1,0)$ and $\tilde{e}_{2}^{\prime \prime}=(0,1)$. Then these cells of $\tilde{X}^{\prime \prime}$ are clearly a $\mathbf{Z}\left[\pi^{\prime \prime}\right]$-basis for the cellular chains group $C_{*}\left(\tilde{X}^{\prime \prime}\right)$ as a $\mathbf{K}\left[\pi^{\prime \prime}\right]$-module. A straightforward calculation shows that

$$
\begin{aligned}
L_{\left(\pi^{\prime \prime}, \varphi^{\prime \prime}\right)} S[X ; f]= & {\left[1 ; \pi^{\prime \prime}\right]_{\varphi^{\prime \prime}}-\left(\left[1 ; \pi^{\prime \prime}\right]_{\varphi^{\prime \prime}}+\left[\sigma ; \pi^{\prime \prime}\right]_{\varphi^{\prime \prime}}+\left[\sigma^{2} ; \pi^{\prime \prime}\right]_{\varphi^{\prime \prime}}\right) } \\
& -\left(-\left[\tau^{-2} ; \pi^{\prime \prime}\right]_{\varphi^{\prime \prime}}-\left[\tau^{-1}, \pi^{\prime \prime}\right]_{\varphi^{\prime \prime}}\right) .
\end{aligned}
$$

Clearly $L_{\left(\pi^{\prime \prime}, \varphi^{\prime \prime}\right)} S[X, f] \neq 0$ but $L(f)=0$. Note that Corollary (1.10) implies that the generalized Lefschetz number at the universal setting is also $\neq 0$. Theorem (1.13) 
implies that the Nielsen number of $f$ is $\geqslant 4$. To obtain higher dimensional examples, we thicken $X$. The generalized Lefschetz and Nielsen numbers remain the same by virtue of Proposition (1.14) and Theorem (1.13).

EXAMPLE (2.2) (after McCord [5]). The best one could do in the manner of Example (2.1) is to obtain a compact manifold with boundary. To obtain a closed manifold one proceeds differently. Let $\tilde{M}=\mathbf{R}^{2}, \pi=$ the group of translations, and

$$
\tilde{f}: \tilde{M} \rightarrow \tilde{M}
$$

the linear map defined by the matrix $\left(\begin{array}{cc}-1 & -1 \\ 2\end{array}\right)$. Put $M=\tilde{M} / \pi$ and note that $\tilde{f}$ induces a homeomorphism $f: M \rightarrow M$ which fits in the universal $(\pi, \varphi)$-setting

$$
\begin{array}{llll} 
& \tilde{M} & \stackrel{\tilde{f}}{\rightarrow} & \tilde{M} \\
S_{(\pi, \varphi)}[M, f]: & p \downarrow & & \downarrow p \\
& M & \stackrel{f}{\rightarrow} & M
\end{array}
$$

where $\varphi: \pi \rightarrow \pi$ is the homomorphism defined by sending $\sigma$ to $\tau^{2} \sigma^{-1}$ and $\tau$ to $\tau \sigma^{-1}$. If we consider $\tilde{M}$ as a $C W$-complex with the usual subdivision defined by the unit lattice, the vertical and horizontal unit segments and the unit square, we see that $\tilde{f}$ is cellular and a straightforward computation shows that the chain map $C_{*}(\tilde{f})$ : $C_{*} \tilde{M} \rightarrow C_{*} \tilde{M}$ is as described by the formulas

$$
\begin{aligned}
& C_{0}(\tilde{f})\left(\tilde{e}_{0}\right)=\tilde{e}_{0}, \\
& C_{1}(\tilde{f})\left(\tilde{e}_{1}\right)=-\left(\tilde{e}_{1}\right) \tau^{2} \sigma^{-1}+\tilde{e}_{1}^{\prime}(1+\tau), \\
& C_{1}(\tilde{f})\left(\tilde{e}_{1}^{\prime}\right)=-\left(\tilde{e}_{1}\right)\left(\sigma^{-1}\right)+\tilde{e}_{1}^{\prime}\left(\sigma^{1}\right), \\
& C_{2}(\tilde{f})\left(\tilde{e}_{2}\right)=\tilde{e}_{2}\left(\sigma^{-1}+\sigma^{-1} \tau-\sigma^{-2} \tau^{2}\right) .
\end{aligned}
$$

Here $\tilde{e}_{0}=(0,0), \tilde{e}_{1}=(1,0), \tilde{e}_{1}^{\prime}=(0,1)$ and $\tilde{e}_{2}$ is the unit square. We also have, by easy calculations, that

$$
\begin{aligned}
R_{\varphi}[\pi] \cong\{[1],[\tau]\} \cong \mathbf{Z}_{2}, \\
{\left[\tau^{2} \boldsymbol{\sigma}^{-1}\right]=[1]=\left[\sigma^{-1}\right]=[\boldsymbol{\sigma}]=\left[\tau^{2} \boldsymbol{\sigma}^{-2}\right], } \\
{\left[\tau \boldsymbol{\sigma}^{-1}\right]=[\tau] . }
\end{aligned}
$$

So we conclude that

$$
L_{(\pi, \varphi)} S[M, f]=[1]+[\tau] .
$$

(Let us note here that this calculation can be avoided by appealing directly to Corollary (1.9): take $\pi^{\prime}=\operatorname{image}(\varphi-1)$ and note that $\left[\pi: \pi^{\prime}\right]=2$. Also the conditions of the corollary are satisfied because $\pi$ is abelian and $\tilde{M}$ is contractible.)

Since $L(f) \neq 0$ we need to alter $M$ in such a way that one of the two terms in $L_{(\pi, \varphi)} S[M, f]$ is reversed in sign. In order to do so, we note that $f$ has two fixed points $x_{0}=p((0,0))$ and $x_{1}=p\left(\left(\frac{1}{2}, 0\right)\right)$. Also, according to Theorem (1.13), $x_{0}$ corresponds to the $(\pi, \varphi)$-Reidemeister class $[1 ; \pi]_{\varphi}$ and $x_{1}$ to $[1, \tau]_{\varphi}$. Now by standard techniques, say, the Tubular Neighborhood Theorem, we can modify $f$ by an isotopy to obtain a diffeomorphism

$$
g:\left(M ; D^{2}, M-D^{2}\right) \rightarrow\left(M ; D^{2}, M-D^{2}\right)
$$


where $D^{2}$ is a small disk centered at $x_{1}$. Since the local index of $f$ at $x_{1}$ is 1 , we can assume that $g$ is actually fixed on $D^{2}$. Now by definition, let

$$
h: M \sharp M \rightarrow M \sharp M
$$

be equal to $g$ on the first summand and to the identity on the second. Let

$$
\tilde{M}-\amalg \tilde{D}^{2}=p^{-1}\left(M-D^{2}\right)
$$

where $p: \tilde{M} \rightarrow M$ is the projection in the setting $S_{(\pi, \varphi)}[M, f]$, and define $(\widetilde{M \sharp M})^{\prime \prime}$ to be the cover of $M \# M$ obtained by attaching to $\tilde{M}-\amalg \tilde{D}^{2}$ copies of $M-D^{2}$ along the circles in $\tilde{M}-\amalg \tilde{D}^{2}$ which form the boundaries of the deleted disks $\amalg \tilde{D}^{2}$. Thus we obtain a setting

$$
\begin{array}{cccc} 
& (\widetilde{M \# M})^{\prime \prime} & \rightarrow & (\widetilde{M \# M})^{\prime \prime} \\
S_{(\pi, \varphi)}[M \sharp M, h]: & \downarrow p^{\prime \prime} & & \downarrow p^{\prime \prime} \\
& M \sharp M & \stackrel{h}{\rightarrow} & M \sharp M
\end{array}
$$

where $p^{\prime \prime}$ is equal to $p$ on $\tilde{M}-\amalg \tilde{D}^{2}$ and to the identity on each of the attached copies $M-D^{2}$. Finally we find that $L_{(\pi, \varphi)} S[M \sharp M, h]=[1]-[\tau]$ since the generalized Lefschetz number of the setting restricted to the first factor is [1], while it is $-[\tau]$ when restricted to the second factor and 0 on the intersection. Hence if $S_{(\gamma, \psi)}[M \sharp M, h]$ is the universal setting for $h: M \sharp M \rightarrow M \sharp M$, Corollary (1.10) implies that $L_{(\gamma, \psi)} S[M \sharp M, h] \neq 0$. Clearly, the ordinary Lefschetz number $L(f)$ is 0.

EXAMPLE (2.3). To obtain higher-dimensional examples, one takes

$$
h \times 1:(M \sharp M) \times S^{n} \rightarrow(M \# M) \times S^{n}, \quad n \geqslant 2,
$$

where $M \# M$ and $h: M \sharp M \rightarrow M \# M$ are as in Example (2.2). But $M \sharp M$ has a complicated fundamental group and, therefore, $L_{(\gamma, \psi)} S[M \sharp M, h]$, is hard to calculate. However, it is possible to modify $M$ to make the ordinary Lefschetz number 0 without changing the fundamental group as follows. First, by definition and keeping the notation of Example (2.2), let

$$
f \times r: M \times S^{n} \rightarrow M \times S^{n}
$$

where $n$ is even and $\geqslant 2$, and $r: S^{n} \rightarrow S^{n}$ is a notation with the north and south poles as the only fixed points. Then it can be easily proved that

$$
L_{(\pi, \varphi)} S\left[M \times S^{n}, f \times r\right]=2[1]+2[\tau]
$$

where $S_{(\pi, \varphi)}\left[M \times S^{n}, f \times r\right]$ is the $(\pi, \varphi)$-setting obtained by multiplying $S_{(\pi, \varphi)}[M, f]$ by $S^{n}$. Observe that $f \times r$ has exactly four fixed points $\left(x_{0}, s_{0}\right),\left(x_{0}, s_{1}\right)$, $\left(x_{1}, s_{0}\right)$ and $\left(x_{1}, s_{1}\right)$ where $x_{0}, x_{1}$ are the fixed points of $f: M \rightarrow M$ while $s_{0}, s_{1}$ are the fixed points of $r: S^{n} \rightarrow S^{n}$. Note also that $\left(x_{1}, s_{0}\right)$ and $\left(x_{1}, s_{1}\right)$ correspond to $[\tau]$. Now proceeding as in Example (2.2), let $D^{\prime}, D^{\prime \prime}$ be two disjoint and sufficiently small $(n+2)$-dimensional disks centered at $\left(x_{1}, s_{0}\right)$ and $\left(x_{1}, s_{1}\right)$, respectively, and modify $f \times r$ by a diffeotopy which is fixed outside a neighborhood of $D^{\prime} \amalg D^{\prime \prime}$ which excludes the other fixed points to obtain a diffeomorphism

$$
\begin{aligned}
g^{\prime}:\left(M \times S^{n} ; D^{\prime} \amalg D^{\prime \prime}, M \times S^{n}-\left(D^{\prime} \amalg D^{\prime \prime}\right)\right) \\
\rightarrow\left(M \times S^{n} ; D^{\prime} \amalg D^{\prime \prime}, M \times S^{n}-\left(D^{\prime} \amalg D^{\prime \prime}\right)\right)
\end{aligned}
$$


which takes $D^{\prime}$ to $D^{\prime}$ and $D^{\prime \prime}$ to $D^{\prime \prime}$. We can also assume that $g \mid\left(D^{\prime} \amalg D^{\prime \prime}\right)$ is the identity. Next let $N^{\prime}, N^{\prime \prime}$ be two copies of a simply connected manifold of dimension $n+2$ and Euler characteristic 0 , and choose two $(n+2)$-dimensional disks $\overline{D^{\prime}} \subset N^{\prime}$, $\bar{D}^{\prime \prime} \subset N^{\prime \prime}$. By definition, let

$$
M^{\prime}=\left(M \times S^{n}-\left(D^{\prime} \amalg D^{\prime \prime}\right)\right) \cup\left(\left(N^{\prime}-\overline{D^{\prime}}\right) \amalg\left(N^{\prime \prime}-\overline{D^{\prime \prime}}\right)\right)
$$

be the orientable manifold obtained by identifying $\partial D^{\prime}$ with $\partial{\overline{D^{\prime}}}^{\prime}$ and $\partial D^{\prime \prime}$ with $\partial{\overline{D^{\prime \prime}}}^{\prime}$, and let $h^{\prime}: M^{\prime} \rightarrow M^{\prime}$ be the map equal to $g^{\prime}$ on the first summand and to the identity on the others. Then

$$
L_{(\pi, \varphi)} S\left[M^{\prime}, h^{\prime}\right]=2[1]-2[\tau]
$$

where $S_{(\pi, \varphi)}\left[M^{\prime}, h^{\prime}\right]$ is the $(\pi, \varphi)$-setting defined by the universal cover of $M^{\prime}$. Clearly, the ordinary Lefschetz number $L\left(h^{\prime}\right)$ of $h^{\prime}$ is 0 . Note that $h^{\prime}$ has exactly two essential Nielsen classes of fixed points and as $\operatorname{dim} M^{\prime}>3$, it is homotopic to a map with exactly two fixed points corresponding to the two essential Nielsen classes.

3. Proofs of Propositions (1.2), (1.3) and (1.4). Suppose that $\varphi_{1}:\left(\pi_{1}, \pi_{1}^{\prime}\right) \rightarrow\left(\pi_{2}, \pi_{2}^{\prime}\right)$ and $\varphi_{2}:\left(\pi_{2}, \pi_{2}^{\prime}\right) \rightarrow\left(\pi_{1}, \pi_{1}^{\prime}\right)$ are homomorphisms of groups. The following proposition is the key to much of what follows.

Proposition (3.1). Suppose that $\left(P_{1}, P_{1}^{\prime}\right)$ and $\left(P_{2}, P_{2}^{\prime}\right)$ are, respectively, a finitelygenerated free $\pi_{1}^{\prime}$-based right $\mathbf{K}\left[\pi_{1}\right]$-module, and a $\pi_{2}^{\prime}$-based right $\mathrm{K}\left[\pi_{2}\right]$-module, and let $f: P_{1} \rightarrow P_{2}$ and $g: P_{1} \rightarrow P_{2}$ be, respectively, $a\left(\pi_{1}, \varphi_{1}\right)$-homomorphism and $a\left(\pi_{2}, \varphi_{2}\right)$ homomorphism. Assume also that $f\left(P_{1}^{\prime}\right) \subset P_{2}^{\prime}$. Then

$$
\left(\varphi_{1}\right)_{*} \operatorname{tr}_{\left(\pi_{1}^{\prime}, \varphi_{2} \circ \varphi_{1}\right)}\left[P_{1}, P_{1}^{\prime} ; g \circ f\right]=\operatorname{tr}_{\left(\pi_{2}^{\prime}, \varphi_{1} \circ \varphi_{2}\right)}\left[P_{2}, P_{2}^{\prime} ; f \circ g\right]
$$

where

$$
\left(\varphi_{1}\right)_{*}: \mathbf{K} R_{\varphi_{2} \circ \varphi_{1}}\left[\pi_{1}, \pi_{1}^{\prime}\right] \rightarrow \mathbf{K} R_{\varphi_{1} \circ \varphi_{2}}\left[\pi_{2}, \pi_{2}^{\prime}\right]
$$

is the homomorphism induced by $\varphi_{1}$.

The proof is just a straightforward computation. Let $\{x\}$ be a $\pi_{1}^{\prime}$-basis for $P_{1}^{\prime}$, and $\{y\}$ a $\pi_{2}^{\prime}$-basis for $P_{2}^{\prime}$. Then, by definition, $\{x\}$ is a $\pi_{1}$-basis for $P_{1}$, and $\{y\}$ a $\pi_{2}$-basis for $P_{2}$. Note that $f: P_{1} \rightarrow P_{1}$ is represented by a matrix $A$ with entries in $\mathbf{K}\left[\pi_{2}^{\prime}\right]$, since $f\left(P_{1}^{\prime}\right) \subset P_{2}^{\prime}$, while $g: P_{2} \rightarrow P_{1}$ is represented by a matrix $B$ with entries in $\mathbf{K}\left[\pi_{1}\right]$. A direct and simple computation shows that $g \circ f: P_{1} \rightarrow P_{1}$ is represented by $B A^{\varphi_{2}}$ and $f \circ g: P_{2} \rightarrow P_{2}$ by $A B^{\varphi_{1}}$ where $(\cdot)^{\varphi}$ is obtained from the matrix $(\cdot)$ by applying $\varphi$ to each of its entries (cf. Proposition (1.1) (2)). Now, by definition, we have

$$
\operatorname{tr}_{\left(\pi_{1}^{\prime}, \varphi_{2} \circ \varphi_{1}\right)}\left[P_{2}, P_{2}^{\prime}: g \circ f\right]=\operatorname{tr}_{\left(\pi_{1}^{\prime} ; \varphi_{2} \circ \varphi_{1}\right)}\left(B A^{\varphi_{2}}\right)
$$

and

$$
\operatorname{tr}_{\left(\pi_{2}^{\prime}, \varphi_{1} \circ \varphi_{2}\right)}\left[P_{2}, P_{2}^{\prime} ; f \circ g\right]=\operatorname{tr}_{\left(\pi_{2}^{\prime}, \varphi_{1} \circ \varphi_{2}\right)}\left(A B^{\varphi_{1}}\right)
$$

But

$$
\begin{aligned}
\left(\varphi_{1}\right)_{*} \operatorname{tr}_{\left(\pi_{1}, \varphi_{2} \circ \varphi_{1}\right)}\left(B A^{\varphi_{2}}\right) & =\operatorname{tr}_{\left(\pi_{2}^{\prime}, \varphi_{1} \circ \varphi_{2}\right)}\left(\left(B A^{\varphi_{2}}\right)^{\varphi_{1}}\right) \\
& =\operatorname{tr}_{\left(\pi_{2}^{\prime}, \varphi_{1} \circ \varphi_{2}\right)}\left(B^{\varphi_{1}} A^{\varphi_{1} \circ \varphi_{2}}\right) .
\end{aligned}
$$


Finally, the commutativity of $\operatorname{tr}_{\left(\pi_{2}^{\prime}, \varphi_{1} \circ \varphi_{2}\right)}$ implies that

$$
\operatorname{tr}_{\left(\pi_{2}^{\prime}, \varphi_{1} \circ \varphi_{2}\right)}\left(B^{\varphi_{1}} A^{\varphi_{1} \circ \varphi_{2}}\right)=\operatorname{tr}_{\left(\pi_{2}^{\prime}, \varphi_{1} \circ \varphi_{2}\right)}\left(A B^{\varphi_{1}}\right) .
$$

This proves the proposition.

Now let $\varphi: \pi \rightarrow \pi$ be a homomorphism, $\pi^{\prime} \subset \pi$ a subgroup, and $\left[P, P^{\prime} ; f\right]$ a finitely-generated, $\pi^{\prime}$-based and $\mathbf{K}[\pi]$-projective module. We would like to define $\operatorname{tr}_{\left(\pi^{\prime}, \varphi\right)}\left[P, P^{\prime} ; f\right]$, when $P^{\prime}$ is not necessarily free. Choose a finitely-generated right $\pi^{\prime}$-projective module $Q^{\prime}$ so that $F^{\prime}=P^{\prime} \oplus Q^{\prime}$ is $\mathbf{K}\left[\pi^{\prime}\right]$-free. If we let $F=F^{\prime}$ $\otimes_{\mathbf{K}\left[\pi^{\prime}\right]} \mathbf{K}[\pi]$, we see that $\left(F, F^{\prime}\right)$ is a free finitely-generated $\pi^{\prime}$-based $\mathbf{K}[\pi]$-module, and that $F=P \oplus Q$ where $Q=Q^{\prime} \otimes_{\mathbf{K}\left[\pi^{\prime}\right]} \mathbf{K}[\pi]$ is a finitely-generated projective $\mathbf{K}[\pi]$-module. Let $O_{Q}: Q \rightarrow Q$ be the zero homomorphism and, by definition, put

$$
\operatorname{tr}_{\left(\pi^{\prime}, \varphi\right)}\left[P, P^{\prime} ; f\right]=\operatorname{tr}_{\left(\pi^{\prime}, \varphi\right)}\left[F, F^{\prime} ; f \oplus O_{Q}\right] \text {. }
$$

To see that $\operatorname{tr}_{\left(\pi^{\prime} ; \varphi\right)}\left[P, P^{\prime} ; f\right]$ is well defined (compare [6]), suppose that $Q_{1}^{\prime}$ is another finitely-generated right $\mathbf{K}\left[\pi^{\prime}\right]$-projective module such that $F_{1}^{\prime}=P^{\prime} \oplus Q_{1}^{\prime}$ is $\pi^{\prime}$-free. Denote $F_{1}^{\prime} \otimes_{\mathbf{K}\left[\pi^{\prime}\right]} \mathbf{K}[\pi]$ by $F_{1}$ and $Q_{1}^{\prime} \otimes_{\mathbf{K}\left[\pi^{\prime}\right]} \mathbf{K}[\pi]$ by $Q_{1}$. We need show that

$$
\operatorname{tr}_{\left(\pi^{\prime}, \varphi\right)}\left[F, F^{\prime} ; f+O_{Q}\right]=\operatorname{tr}_{\left(\pi^{\prime}, \varphi\right)}\left[F_{1}, F_{1}^{\prime} ; f \oplus O_{Q_{1}^{\prime}}\right] \text {. }
$$

First of all note that the additivity property of $\operatorname{tr}_{\left(\pi^{\prime}, \varphi\right)}$ for maps of $\pi^{\prime}$-based $\mathbf{K}[\pi]$-modules implies that

$$
\operatorname{tr}_{\left(\pi^{\prime} ; \varphi\right)}\left[F, F^{\prime} ; f \oplus O_{Q}\right]=\operatorname{tr}_{\left(\pi^{\prime} ; \varphi\right)}\left[F \oplus F_{1} ; F^{\prime} \oplus F_{1}^{\prime} ; f \oplus O_{Q} \oplus O_{F_{1}}\right]
$$

and

$$
\operatorname{tr}_{\left(\pi^{\prime}, \varphi\right)}\left[F_{1}, F_{1}^{\prime} ; f \oplus O_{Q_{1}}\right]=\operatorname{tr}_{\left(\pi^{\prime} ; \varphi\right)}\left[F_{1} \oplus F, F_{1}^{\prime} \oplus F^{\prime} ; f \oplus O_{Q_{1}} \oplus O_{F}\right]
$$

where $f \oplus O_{Q} \oplus O_{F_{1}}: F \oplus F_{1} \rightarrow F \oplus F_{1}$ and $f \oplus O_{Q} \oplus O_{F}: F_{1} \oplus F \rightarrow F_{1} \oplus F$. But,

$$
\begin{aligned}
F^{\prime} \oplus F_{1}^{\prime} & =\left(P^{\prime} \oplus Q^{\prime}\right) \oplus\left(P^{\prime} \oplus Q_{1}^{\prime}\right) \\
& \cong\left(P^{\prime} \oplus\left(Q^{\prime} \oplus\left(P^{\prime} \oplus Q_{1}^{\prime}\right)\right)\right) \\
& \cong\left(P^{\prime} \oplus\left(Q_{1}^{\prime} \oplus\left(P^{\prime} \oplus Q^{\prime}\right)\right)\right) \\
& \cong\left(P^{\prime} \oplus Q_{1}^{\prime}\right) \oplus\left(P^{\prime} \oplus Q^{\prime}\right) \\
& =F_{1}^{\prime} \oplus F^{\prime}
\end{aligned}
$$

where all the isomorphisms are all $\pi^{\prime}$-isomorphisms leaving the first summand $\boldsymbol{P}^{\prime}$ fixed. Hence there is a $\mathbf{K}\left[\pi^{\prime}\right]$-isomorphism

$$
\alpha: F^{\prime} \oplus F_{1}^{\prime} \rightarrow F_{1}^{\prime} \oplus F^{\prime}
$$

such that $\alpha \mid P^{\prime}=1_{P^{\prime}}$. Denote the natural extension to $F \oplus F_{1}$ also by $\alpha$ and note that

$$
f \oplus O_{Q} \oplus O_{F_{1}}=\alpha^{-1}\left(f \oplus O_{Q_{1}} \oplus O_{F}\right) \alpha
$$

Proposition (3.1) now applies and yields the equality

$$
\operatorname{tr}_{\left(\pi^{\prime}, \varphi\right)}\left[F \oplus F_{1}, F^{\prime} \oplus F_{1}^{\prime} ; f \oplus O_{Q} \oplus O_{F_{1}}\right]=\operatorname{tr}_{\left(\pi^{\prime}, \varphi\right)}\left[F_{1} \oplus F, F_{1}^{\prime} \oplus F^{\prime} ; f \oplus O_{Q_{1}} \oplus O_{F}\right]
$$

This equality taken with the two equalities at the beginning of the argument give the desired result. 
It is quite easy to show that Proposition (3.1) remains valid in the more general case when $\left(P_{1}, P_{1}^{\prime}\right)$ and $\left(P_{2}, P_{2}^{\prime}\right)$ are projective but not necessarily free. Proposition (1.2) is now an immediate consequence of Proposition (3.1).

Proof of Proposition (1.3). Suppose that $[C ; f]$ and $[C ; g]$ are finitely-generated $\mathbf{K}[\pi, \varphi]$-projective chain complexes, and let

$$
\delta: C \rightarrow C
$$

be a $(\pi, \varphi)$-map such that

$$
\delta \partial+\partial \delta=f-g .
$$

The proof is formally the same as that of the corresponding assertion in [6]. We have

$$
L_{(\pi, \varphi)}[C ; f-g]=L_{(\pi, \varphi)}[C ; \delta \partial]+L_{(\pi, \varphi)}[C ; \partial \delta] .
$$

Now if we apply Proposition (1.2) to replace $\delta \partial$ by $\partial \delta$ in each of the summands of the alternating sum defining $L_{(\pi, \varphi)}[C ; \delta \partial]$ one sees immediately the two terms above cancel each other to yield the desired result.

We shall need a slightly more general form of the Homotopy Invariance. Suppose that $\left[C_{1} ; f_{1}\right]$ and $\left[C_{2} ; f_{2}\right]$ are two finitely-generated $\mathbf{K}[\pi, \varphi]$-projective complexes. Assume that

$$
\begin{array}{ccc}
C_{1} & \stackrel{i}{\rightarrow} & C_{2} \\
f_{1} \downarrow & & \downarrow f_{2} \\
C_{1} & \stackrel{i}{\rightarrow} & C_{2}
\end{array}
$$

commutes up to a $(\pi, \varphi)$-chain homotopy, where $i$ is a $\pi$-chain map.

Proposition (3.2). Suppose that the induced homomorphism

$$
H_{*}(i): H_{*} C_{1} \rightarrow H_{*} C_{2}
$$

is an isomorphism. Then

$$
L_{(\pi, \varphi)}\left[C_{1} ; f_{1}\right]=L_{(\pi, \varphi)}\left[C_{2} ; f_{2}\right]
$$

Proof. Using the mapping cone of $i: C_{1} \rightarrow C_{2}$ we can find $\pi$-maps $r: C_{2} \rightarrow C_{1}, \delta_{1}$ : $C_{1} \rightarrow C_{1}, \delta_{2}: C_{2} \rightarrow C_{2}$ such that $r$ is a $\pi$-chain map and

$$
\delta_{1} \partial_{1}+\partial_{1} \delta_{1}=\mathrm{id}_{1}-r i, \quad \delta_{2} \partial_{2}+\partial_{2} \delta_{2}=\mathrm{id}_{2}-i r
$$

where $\mathrm{id}_{1}: C_{1} \rightarrow C_{1}, \mathrm{id}_{2}: C_{2} \rightarrow C_{2}$ are the identity maps. Now note that $r f_{2} i$ and $r i f_{1}$ are also $(\pi, \varphi)$-chain homotopic and hence

$$
L_{\left(\pi^{\prime}, \varphi\right)}\left[C_{1} ; r f_{2} i\right]=L_{\left(\pi^{\prime}, \varphi\right)}\left[C_{1} ; r i f_{1}\right]
$$

by Proposition (1.3). Next observe that

$$
L_{\left(\pi^{\prime}, \varphi\right)}\left[C_{1} ; r f_{2} i\right]=L_{\left(\pi^{\prime}, \varphi\right)}\left[C_{2} ; i r f_{2}\right]
$$

by Proposition (1.2). But by Proposition (1.3),

$$
L_{\left(\pi^{\prime}, \varphi\right)}\left[C_{2} ; \operatorname{irf}_{2}\right]=L_{\left(\pi^{\prime}, \varphi\right)}\left[C_{2} ; f_{2}\right]
$$

since $\operatorname{irf} f_{2}$ and $f_{2}$ are easily seen to be $(\pi, \varphi)$-chain homotopic. The proposition is therefore proved. 
Proof of Proposition (1.4). Let $j: Z_{*} C \rightarrow H_{*} C$ be the natural projection, with $Z_{*} C$ being the module of cycles. Since $H_{*} C$ is projective, we can find a $\mathbf{K}[\pi]$-homomorphism $j^{-1}: H_{*} C \rightarrow Z_{*} C \subset C_{*}$ such that $j^{-1} \circ j=1$. Note that $f \circ j^{-1}-$ $j^{-1} \circ H_{*} f$ takes $H_{*} C$ to the submodule of boundaries $B_{*} C \subset C_{*}$. By Lemma (3.3), there is a $(\pi, \varphi)$-map $\delta: H_{*} C \rightarrow C_{*}$ such that

$$
\partial \delta=f \circ j^{-1}-j^{-1} \circ H_{*} f
$$

If we regard $H_{*} C$ as a chain complex with the trivial boundary homomorphism, we see that the diagram

$$
\begin{array}{lll}
H_{*} C & \stackrel{j^{-1}}{\rightarrow} & C_{*} \\
H_{*} f \downarrow & & \downarrow f \\
& & \\
H_{*} C & \stackrel{j^{-1}}{\rightarrow} & C_{*}
\end{array}
$$

commutes up to chain homotopy. Hence Proposition (3.2) applies and we obtain the result that

$$
L_{(\pi, \varphi)}[C ; f]=L_{(\pi, \varphi)}\left[H_{*} C ; H_{*} f\right]
$$

as required.

LEMMA (3.3). Let

$$
0 \rightarrow M^{\prime} \stackrel{i}{\rightarrow} M \stackrel{j}{\rightarrow} M^{\prime \prime} \rightarrow 0
$$

be an exact sequence of right $\mathbf{K}[\pi]$-modules and homomorphisms. Suppose that $P$ is a $\mathbf{K}[\pi]$-projective module and assume

$$
f^{\prime \prime}: P \rightarrow M^{\prime \prime}
$$

is $a(\pi, \varphi)$-homomorphism. Then there is $a(\pi, \varphi)$-homomorphism

$$
f: P \rightarrow M
$$

such that $j \circ f=f^{\prime \prime}$.

Proof. Suppose first that $P$ is free and let $\{x\}$ be a $\pi$-basis. Define $f$ on $\{x\}$ so that $j \circ f=f^{\prime \prime}$, and then extend it to a $(\pi, \varphi)$-map. If $P$ is projective then $P$ is a direct summand of a free $\mathbf{K}[\pi]$-module $F$. Then the composite $F \stackrel{r}{\rightarrow} P \stackrel{f^{\prime \prime}}{\rightarrow} M^{\prime \prime}$, where $r$ is a retraction on $P$, is an extension of $f^{\prime \prime}$ to all of $F$. Since $F$ is free, $f^{\prime \prime} r$ can be lifted to $M$. Now let $f$ be the restriction of this lift to $P$.

4. Proof of Theorem (1.5) and Corollary (1.6). Suppose that $\pi^{\prime} \subset \pi$ is a subgroup with finite index $\left[\pi: \pi^{\prime}\right]<\infty$ and such that $\varphi\left(\pi^{\prime}\right) \subset \pi^{\prime}$. To prove the theorem it suffices to consider the case when the graded module is free and concentrated in a single dimension. So suppose that $P$ is a finitely-generated free right $\mathbf{K}[\pi]$-module, and let $f: P \rightarrow P$ be a $(\pi, \varphi)$-map. Choose a $\pi$-basis $\{x\}$ for $P$, and denote by $P_{x}$ the free $\pi$-module generated by the element $x$ of the basis. Then if $i_{x}: P_{x} \rightarrow P$ is the natural imbedding, and $j_{x}: P \rightarrow P_{x}$ the natural projection, we see that

$$
\operatorname{tr}_{(\pi, \varphi)}[P ; f]=\sum_{x} \operatorname{tr}_{(\pi, \varphi)}\left[P_{x} ; j_{x} f i_{x}\right]
$$


and

$$
\operatorname{tr}_{\left(\pi^{\prime}, \varphi\right)}[P ; f]=\sum_{x} \operatorname{tr}_{\left(\pi^{\prime}, \varphi\right)}\left[P_{x} ; j_{x} f i_{x}\right]
$$

with $x$ ranging over the basis $\{x\}$. Hence it suffices to prove Theorem (1.5) when $P$ is generated by a single element. So consider the equation

$$
f(x)=x \cdot \lambda
$$

where

$$
\lambda=\sum \lambda_{\sigma} \sigma
$$

is an element of $\mathbf{K}[\pi]$. Now let $\alpha \in \pi^{\prime}$, and write (4.2) in the form

$$
\lambda=\sum_{\beta} \lambda_{\beta} \beta+\lambda^{\prime}
$$

where $\beta$ ranges over the set of elements of the class $[\alpha]=[\alpha ; \pi]_{\varphi}$, and $\lambda^{\prime}$ is a linear combination in the elements of $\pi$ not in $[\alpha ; \pi]_{\varphi}$. Let $S=\{\sigma\}$ be a set of representatives of the cosets of $\pi \bmod \pi_{\alpha}$, where

$$
\pi_{\alpha}=\left\{\rho \in \pi \mid \rho \alpha \varphi(\rho)^{-1}=\alpha\right\} .
$$

Thus we see that

$$
\lambda_{[\alpha ; \pi]_{\varphi}}=\sum_{\beta \in[\alpha]} \lambda_{\beta}=\sum_{\sigma \in S} \lambda_{\sigma \alpha \varphi(\sigma)^{-1}}
$$

where $\lambda_{[\alpha ; \pi]_{\varphi}}$ is the coefficient of $[\alpha ; \pi]_{\varphi}$ in $L_{(\pi, \varphi)}[P ; f]$. Next let $\pi_{\alpha}^{\prime}=\pi_{\alpha} \cap \pi^{\prime}$, and choose a set of representatives $T=\{\tau\}$ of the cosets of $\pi_{\alpha} \bmod \pi_{\alpha}^{\prime}$. The assumption that $\left[\pi: \pi^{\prime}\right]$ is finite implies that $\left[\pi_{\alpha}: \pi_{\alpha}^{\prime}\right]$ is also finite, and we have

$$
\left[\pi_{\alpha}: \pi_{\alpha}^{\prime}\right] \cdot \lambda_{[\alpha ; \pi]_{\varphi}}=\sum_{\sigma \in S}\left(\sum_{\tau \in T} \lambda_{\sigma \tau \alpha \varphi(\sigma \tau)^{-1}}\right)=\sum_{\sigma \tau} \lambda_{\sigma \tau \alpha \varphi(\sigma \tau)^{-1}}
$$

where $\sigma \tau$ ranges over a set of coset representatives of $\pi \bmod \pi_{\alpha}^{\prime}$, for

$$
\pi=\underset{\sigma}{\amalg} \sigma \pi_{\alpha}=\underset{\sigma}{\amalg}\left(\sigma \underset{\tau}{\amalg} \tau \pi_{\alpha}^{\prime}\right)=\underset{\sigma \tau}{\amalg} \sigma \tau \pi_{\alpha}^{\prime} .
$$

Now let $\Xi_{\alpha}=\{\xi\}$ be a set of coset representatives of $\pi^{\prime} \bmod \pi_{\alpha}^{\prime}$, and $Z=\{\zeta\}$ a set of coset representatives of $\pi \bmod \pi^{\prime}$. Then $\{\zeta \xi\}$ is a set of coset representatives of $\pi \bmod \pi_{\alpha}^{\prime}$, and we can rewrite (4.4) in the form

$$
\left[\pi_{\alpha}: \pi_{\alpha}^{\prime}\right] \lambda_{[\alpha ; \pi]_{\varphi}}=\sum_{\zeta \xi \in Z \Xi_{\alpha}} \lambda_{\zeta \xi \alpha \varphi(\zeta \xi)^{-1}}=\sum_{\zeta \in Z}\left(\sum_{\xi \in \Xi_{\alpha}} \lambda_{\zeta \xi \alpha \varphi(\zeta \xi)^{-1}}\right) .
$$

To finish the proof of the theorem, it suffices to show that the expression on the right is exactly $\lambda_{\left[\alpha ; \pi^{\prime}\right]_{\varphi}}$, the coefficient of $\left[\alpha ; \pi^{\prime}\right]_{\varphi}$ in $L_{\left(\pi^{\prime}, \varphi\right)}[P ; f]$. So first note that $\{x \zeta\}$ with $\zeta \in Z$ is a $\pi^{\prime}$-basis for $P$. Hence to compute $L_{\left(\pi^{\prime}, \varphi\right)}[P ; f]$ in terms of $\{x \zeta\}$, we consider the equation

$$
\begin{aligned}
f(x \zeta) & =(x \zeta) \zeta^{-1} \lambda \varphi(\zeta) \\
& =(x \zeta) \zeta^{-1}\left(\sum_{\beta} \lambda_{\beta} \beta\right) \varphi(\zeta)+x \zeta \cdot \zeta^{-1} \lambda^{\prime} \varphi(\zeta)
\end{aligned}
$$


where $\beta$ ranges over $[\alpha ; \pi]_{\varphi}$ while $\lambda^{\prime}$ is a linear combination in elements $\beta^{\prime}$ not in $[\alpha ; \pi]_{\varphi}$. Hence the contribution of $x \zeta$ to the coefficient $\lambda_{\left[\alpha ; \pi^{\prime}\right]_{\varphi}}$ is exactly the sum $\Sigma_{\beta} \lambda_{\beta}$, where $\beta$ ranges over the set

$$
\zeta\left[\alpha ; \pi^{\prime}\right]_{\varphi} \varphi(\zeta)^{-1}=\zeta\left\{\xi \alpha \varphi(\xi)^{-1} \mid \xi \in \Xi_{\alpha}\right\} \varphi(\zeta)^{-1}
$$

with $\Xi_{\alpha}$ being the set of coset representatives of $\pi^{\prime} \bmod \pi_{\alpha}^{\prime}$ as above. But

$$
\zeta\left\{\xi \alpha \varphi(\xi)^{-1} \mid \xi \in \Xi_{\alpha}\right\} \varphi(\zeta)^{-1}=\left\{\zeta \xi \alpha \varphi(\zeta \xi)^{-1} \mid \xi \in \Xi_{\alpha}\right\}
$$

Therefore the contribution of $x \zeta$ to $\lambda_{\left[\alpha ; \pi^{\prime}\right]_{\Phi}}$ is exactly

$$
\sum_{\xi \in \Xi_{\alpha}} \lambda_{\zeta \xi \alpha \varphi(\zeta \xi)^{-1}}
$$

the $\zeta$ th term in (4.5). Adding up the contributions of all the elements of $\{x \zeta\}$ we see that

$$
\lambda_{\left[\alpha ; \pi^{\prime}\right]_{\varphi}}=\sum_{\zeta}\left(\sum_{\xi} \lambda_{\zeta \xi \alpha \varphi(\zeta \xi)^{-1}}\right)=\left[\pi_{\alpha}: \pi_{\alpha}^{\prime}\right] \lambda_{[\alpha ; \pi]_{\varphi}},
$$

where $\xi$ ranges over $\Xi_{\alpha}$, the set of coset representatives of $\pi^{\prime} \bmod \pi_{\alpha}^{\prime}$, and $\zeta$ over $Z$, the set of coset representatives of $\pi \bmod \pi^{\prime}$. Hence the theorem is proved.

Proof of Corollary (1.6). Suppose that $C$ is a finitely-generated $\mathbf{K}[\pi]$-projective graded complex and assume that $H_{*} C \cong \mathbf{Z}$. Let $f: C \rightarrow C$ be a $(\pi, \varphi)$-chain map such that $L_{(\pi, \varphi)}[C ; f] \neq 0$, and suppose that $[\alpha ; \pi]_{\varphi}$ is an essential class such that $[\pi$ : Fix $\varphi_{\alpha}$ ] is finite, where $\varphi_{\alpha}(\cdot)=\alpha \varphi(\cdot) \alpha^{-1}$. Denote Fix $\varphi_{\alpha}$ by $\pi^{\prime}$, and note that the correspondence $\gamma \mapsto \gamma \alpha^{-1}$ induces an isomorphism $K R_{\varphi}[\pi] \rightarrow K R_{\varphi_{\alpha}}[\pi]$ which takes $L_{(\pi, \varphi)}[C ; f]$ to $L_{\left(\pi, \varphi_{\alpha}\right)}\left[C ; f_{\alpha}\right]$ where $f_{\alpha}(\cdot)=f(\cdot) \alpha^{-1}$. Moreover $[1 ; \pi]_{\varphi_{\alpha}}$ is a $\left(\pi, \varphi_{\alpha}\right)-$ essential class for $f_{\alpha}: C \rightarrow C$. Now by Theorem (1.5) we see that $\left[1 ; \pi^{\prime}\right]_{\varphi_{\alpha}}$ is also $\left(\pi^{\prime}, \varphi_{\alpha}\right)$-essential. Note that $\varphi_{\alpha} \mid \pi^{\prime}=$ identity. Hence $\left[1 ; \pi^{\prime}\right]_{\varphi_{\alpha}}$ is a single element $\{1\}$. If $\zeta \in \operatorname{cent}\left(\operatorname{im} \varphi_{\alpha}\right) \cap \pi^{\prime}$ then

$$
\varphi_{\zeta \alpha}(\cdot)=\zeta \alpha \varphi(\cdot) \alpha^{-1} \zeta^{-1}=\zeta \varphi_{\alpha}(\cdot) \zeta^{-1}=\varphi_{\alpha}(\cdot)
$$

and since $C$ is acyclic, and $f_{\zeta \alpha}$ and $f_{\alpha}$ induces the same homomorphism on $\mathbf{Z}$, it follows that $f_{\zeta \alpha}$ and $f_{\alpha}$ are $\left(\pi^{\prime}, 1\right)$-chain homotopic, and hence

$$
L_{\left(\pi^{\prime}, 1\right)}\left[C ; f_{\zeta \alpha}\right]=L_{\left(\pi^{\prime}, 1\right)}\left[C ; f_{\alpha}\right] .
$$

But the correspondence $\gamma \mapsto \gamma \zeta^{-1}$ induces an isomorphism $\mathbf{Z} R_{\varphi_{\alpha}}\left[\pi^{\prime}\right]=\mathbf{Z} R_{\varphi_{\zeta \alpha}}\left[\pi^{\prime}\right] \rightarrow$ $\mathbf{Z} R_{\varphi_{\alpha}}\left[\pi^{\prime}\right]$. Hence $\left[\zeta ; \pi^{\prime}\right]_{\varphi_{\alpha}}$ is also essential and consists of a single element. As there is a finite number of essential classes for $f_{\alpha}: C \rightarrow C$, it follows that $\pi^{\prime} \cap \operatorname{cent}\left(\operatorname{im} \varphi_{\alpha}\right)$ is finite and hence is equal to $\{1\}$.

5. Proofs of Theorems (1.7) and (1.8). Suppose that $\varphi:\left(\pi, \pi^{\prime}\right) \rightarrow\left(\pi, \pi^{\prime}\right)$ is a homomorphism, where $\pi^{\prime}$ is a normal subgroup, and let $\Xi=\{\xi\}$ be a set of coset representatives of $\pi \bmod \pi^{\prime}$. We need prove that

$$
\Xi_{*} L_{\left(\pi^{\prime}, \varphi\right)}\left[C, C^{\prime} ; f\right]=\sum_{\xi} L_{\left(\pi^{\prime}, \varphi_{\xi}\right)}\left[C^{\prime} ; f_{\xi}^{\prime}\right] \otimes \xi
$$


where $\left[C, C^{\prime} ; f\right]$ is a finitely-generated $\mathbf{K}\left[\pi, \pi^{\prime} ; \varphi\right]$-projective graded module. Recall that the isomorphism

$$
\Xi_{*}: \mathbf{K} R_{\varphi}\left[\pi, \pi^{\prime}\right] \rightarrow \sum_{\xi} \mathbf{K} R_{\varphi_{\xi}}\left[\pi^{\prime}\right] \otimes \xi
$$

is induced by sending $\left[\alpha ; \pi, \pi^{\prime}\right]_{\varphi}$ to $\left[\alpha \xi^{-1} ; \pi^{\prime}\right]_{\varphi_{\xi}} \otimes \xi$ where $\alpha \in \pi^{\prime} \xi, \varphi_{\xi}(\cdot)=\xi \varphi(\cdot) \xi^{-1}$ and that $f_{\xi}^{\prime}$ is the composite

$$
C^{\prime} \stackrel{i}{\rightarrow} C \stackrel{f_{\xi}}{\rightarrow} C \stackrel{j}{\rightarrow} C^{\prime}
$$

with $i$ being the natural injection, $f_{\xi}(\cdot)=f(\cdot) \xi^{-1}$, and $j$ the natural projection.

It suffices to prove the assertion when $C^{\prime}$ is concentrated in a single dimension, and freely generated over $\mathbf{K}\left[\pi^{\prime}\right]$ by a single element $x$, say. So let us consider the equation

$$
f(x)=\lambda x
$$

where

$$
\lambda=\sum \lambda_{\sigma} \sigma,
$$

with the coefficients $\lambda_{\sigma}$ being in $\mathbf{K}$. Express $\lambda$ in the form

$$
\lambda=\lambda_{1}+\cdots+\lambda_{\xi}+\cdots
$$

where $\lambda_{\xi}$ is in $\mathbf{K}\left[\pi^{\prime} \xi\right]$. Now suppose that $\xi$ is the unique element in $\Xi$ such that $\left[\alpha ; \pi, \pi^{\prime}\right]_{\varphi} \subset \pi^{\prime} \xi$, and observe that

$$
f_{\xi}^{\prime}(x)=x \lambda_{\xi} \xi^{-1}=\sum_{\alpha^{\prime}} \lambda_{\alpha^{\prime} \xi} \alpha^{\prime}
$$

where $\alpha^{\prime}$ ranges over $\pi^{\prime}$, and

$$
\lambda_{\xi}=\sum_{\alpha^{\prime}} \lambda_{\alpha^{\prime} \xi} \alpha^{\prime} \xi
$$

with the coefficients being in $\mathbf{K}$. Thus we see that

$$
\lambda_{\left[\alpha ; \pi, \pi^{\prime}\right]_{\varphi}}=\sum \lambda_{\alpha^{\prime} \xi}
$$

with $\alpha^{\prime} \xi$ ranging over the elements of the class $\left[\alpha ; \pi, \pi^{\prime}\right]_{\varphi} \subset \pi^{\prime} \xi$, and

$$
\lambda_{\left[\alpha \xi^{-1} ; \pi^{\prime}\right]_{\varphi}}=\sum \lambda_{\alpha^{\prime} \xi}
$$

where $\alpha^{\prime} \xi$ ranges also over $\left[\alpha ; \pi, \pi^{\prime}\right]_{\varphi}$ since $\left[\alpha ; \pi, \pi^{\prime}\right]_{\varphi} \stackrel{\xi^{-1}}{\rightarrow}\left[\alpha \xi^{-1} ; \pi^{\prime}\right]_{\varphi_{\xi}}$ is a bijection. This proves the theorem.

Proof of Theorem (1.8). Suppose that $C$ is a finitely-generated $\mathbf{K}[\pi, \varphi]$-projective graded module. Put $\pi^{\prime \prime}=\pi / \pi^{\prime}$ and assume that $\left|\pi^{\prime \prime}\right|<\infty$. Then $\varphi$ induces a homomorphism $\varphi^{\prime \prime}: \pi^{\prime \prime} \rightarrow \pi^{\prime \prime}$. Let $Z=\{\zeta\}$ be a set of representatives of the cosets of $\pi \bmod \pi^{\prime}$. Let $\Xi$ be another copy of $Z$, and define $\Omega \subset \Xi$ to be a subset such that its image $\Omega^{\prime \prime} \subset \pi^{\prime \prime}$ is a complete set of representatives of the $\left(\pi^{\prime \prime}, \varphi^{\prime \prime}\right)$-classes of $\pi^{\prime \prime}$. Then we can write

$$
\Xi=\underset{\omega \in \Omega}{\mathrm{I}} \Xi_{\omega}
$$


where $\Xi_{\omega}=\left\{\xi \in \Xi \mid \pi^{\prime} \xi=\pi^{\prime}\right\} \omega \varphi(\zeta)^{-1}$, for some $\left.\zeta \in Z\right\}$. Note that the projection $\pi \rightarrow \pi^{\prime \prime}$ takes $\Xi_{\omega}$ onto $\left[\omega^{\prime \prime}, \pi^{\prime \prime}\right]_{\varphi^{\prime \prime}}$. It is easy to see that the partition of $\Xi$ described above, induces naturally the partition

$$
R_{\varphi}\left[\pi, \pi^{\prime}\right]=\underset{\omega}{\amalg}\left(\underset{\xi \in \Xi_{\omega}}{\amalg} R_{\varphi}\left[\pi^{\prime} \xi, \pi^{\prime}\right]\right) .
$$

By definition, let

$$
\Xi_{*}: \mathbf{K} R_{\varphi}\left[\pi, \pi^{\prime}\right] \rightarrow \sum_{\xi \in \Xi} \mathbf{K} R_{\varphi_{\xi}}\left[\pi^{\prime}\right] \otimes \xi
$$

be the isomorphism induced by sending $\gamma$ to $\gamma \xi^{-1}$, where $\xi$ is the unique element in $\Xi$ such that $\gamma \subset \pi_{\xi}^{\prime}$. Clearly $\Xi_{*}$ preserves the decompositions induced by the partition (5.1) of $\Xi$. Consider now the commutative triangle

$$
\begin{array}{ccc}
\mathbf{K} R_{\varphi}\left[\pi, \pi^{\prime}\right] & \stackrel{\Xi}{\rightarrow} & \sum_{\xi} \mathbf{K} R_{\varphi_{\xi}}\left[\pi^{\prime}\right] \otimes \xi \\
q_{*} \searrow & & \swarrow \Xi_{*}^{\prime} \\
& \mathbf{K} R_{\varphi}[\pi] &
\end{array}
$$

where $q_{*}$ is induced by sending $\left[\gamma ; \pi^{\prime}\right]_{\varphi}$ to $[\gamma ; \pi]_{\varphi}$ and $\Xi_{*}^{\prime}$ by sending $(\gamma, \xi)$ to $\gamma \xi$. It is easy to verify that $q_{*}$ takes the decomposition of $\mathbf{K} R_{\varphi}\left[\pi, \pi^{\prime}\right]$ induced by (5.1)' to a decomposition of $\mathbf{K} R_{\varphi}[\pi]$. Thus we can write

$$
L_{(\pi, \varphi)}[C ; f]=\sum_{\omega \in \Omega} L_{(\pi, \varphi)}[C ; f]_{\omega}
$$

where $L_{(\pi, \varphi)}[C ; f]_{\omega}$ is in the component $\mathbf{K} R_{\varphi}[\pi]_{\omega}$ spanned by the image of $\amalg_{\xi \in \Xi_{\omega}} R_{\varphi}\left[\pi^{\prime} \xi, \pi^{\prime}\right]$. It is clear that $\Xi_{*}^{\prime}$ takes $L_{\left(\pi^{\prime}, \varphi_{\xi}\right)}\left[C ; f_{\xi}\right]$ to $\mathbf{K} R_{\varphi}[\pi]_{\omega}$, where $\xi \in \Xi_{\omega}$. Hence to prove Theorem (1.8) it suffices to show that

$$
\left|\pi^{\prime \prime}\right| L_{(\pi, \varphi)}[C ; f]_{\omega}=\Xi_{*}^{\prime}\left(\sum_{\xi} L_{\left(\pi^{\prime}, \varphi_{\xi}\right)}\left[C ; f_{\xi}\right]\right)
$$

where $\xi$ ranges over $\Xi_{\omega}$. We shall first prove $(5.3)_{\omega}$ when $\omega=1$. Choose a $\pi^{\prime}$-based structure $\left[C, C^{\prime} ; f\right]$ on $[C ; f]$ and note that as a result we have the decomposition

$$
C \cong \sum_{\zeta} C^{\prime} \otimes_{\pi^{\prime}} \pi^{\prime} \zeta
$$

Denote $C^{\prime} \otimes_{\pi^{\prime}} \pi^{\prime} \zeta$ by $C_{\zeta}^{\prime}$ and let $i_{\xi}: C_{\xi}^{\prime} \rightarrow C$ be the natural injection of $C_{\xi}^{\prime}$ as the $\zeta$ th summand and $j_{\zeta}: C \rightarrow C_{\zeta}^{\prime}$ be the natural projection of $C$ onto the $\zeta$ th summand. Define $f_{\zeta}^{\prime}: C^{\prime} \rightarrow C^{\prime}$ to be the composite $j f_{\zeta} i$.

LEMMA (5.4).

$$
\zeta_{*}\left(L_{\left(\pi^{\prime}, \varphi\right)}\left[C_{\xi}^{\prime} ; j_{\xi} f i_{\xi}\right]\right)=L_{\left(\pi^{\prime}, \varphi_{\xi}\right)}\left[C^{\prime} ; f_{\xi}^{\prime}\right],
$$

where $\xi=\zeta \varphi\left(\zeta^{-1}\right)$, and $\zeta_{*}$ is the isomorphism $\mathbf{K} R_{\varphi}\left[\pi^{\prime}\right] \rightarrow \mathbf{K} R_{\varphi_{\xi}}\left[\pi^{\prime}\right]$ induced by sending $\gamma$ to $\zeta \gamma \zeta^{-1}$. 
Proof. It is clear that it suffices to consider the case when $C^{\prime}$ is free over $\mathbf{K}\left[\pi^{\prime}\right]$ and is generated by a single element $x$. Consider the equation $f(x)=x \lambda=x \lambda_{1}^{\prime}$ $+\cdots+\lambda_{\sigma}^{\prime}+\cdots$ where $\lambda_{\sigma}^{\prime} \in \mathbf{K}\left[\pi^{\prime} \sigma\right]$. An easy computation yields the equation

$$
\left(j_{\zeta} f i_{\zeta}\right)(x \zeta)=(x \zeta) \cdot \zeta^{-1} \xi \lambda_{\xi} \varphi(\zeta)
$$

where $\xi=\zeta \varphi(\zeta)^{-1}$ and $\lambda_{\xi}^{\prime}=\xi \lambda_{\xi}$ with $\lambda_{\xi} \in \mathbf{K}\left[\pi^{\prime}\right]$. Hence

$$
\operatorname{tr}_{\left(\pi^{\prime}, \varphi\right)}\left[C_{\zeta}^{\prime} ; j_{\zeta} f i_{\zeta}\right]=\left[\zeta^{-1} \xi \lambda_{\xi} \varphi(\zeta) ; \pi^{\prime}\right]_{\varphi} .
$$

On the other hand we find that

$$
f_{\xi}^{\prime}(x)=j\left(f(x) \xi^{-1}\right)=j\left(x \lambda \xi^{-1}\right)=x \cdot\left(\xi \lambda_{\xi} \xi^{-1}\right),
$$

and therefore we have

$$
\operatorname{tr}_{\left(\pi^{\prime}, \varphi\right)}\left[C^{\prime} ; f_{\xi^{\prime}}\right]=\left[\xi \lambda_{\xi} \xi^{-1} ; \pi^{\prime}\right]_{\varphi_{\xi}}
$$

But

$$
\left(\zeta_{*}\right)\left(\zeta^{-1} \xi \lambda_{\xi} \varphi(\zeta)\right)=\zeta \zeta^{-1} \xi \lambda_{\xi} \varphi(\zeta) \zeta^{-1}=\xi \lambda_{\xi} \xi^{-1},
$$

which proves the lemma.

It may happen, of course, that $\zeta \varphi(\zeta)^{-1}=\xi^{\prime}$ is not in the chosen set of representatives $\Xi$, in which case one has to adjust the isomorphism of Lemma (5.4) as follows. Suppose that $\zeta \varphi(\zeta)^{-1}=\xi^{\prime}=\sigma \xi$, with $\xi \in \Xi$ and $\sigma \in \pi^{\prime}$. Then the correspondence which sends $\gamma$ to $\gamma \sigma$ induces an isomorphism

$$
\nu_{\zeta}: \mathbf{K} R_{\varphi_{\xi}}\left[\pi^{\prime}\right] \rightarrow \mathbf{K} R_{\varphi_{\xi}}\left[\pi^{\prime}\right] .
$$

The adjusted Lemma (5.4) asserts that

$$
\nu_{\zeta} \zeta_{*} L_{\left(\pi^{\prime}, \varphi\right)}\left[C_{\zeta}^{\prime} ; j_{\zeta} f i_{\zeta}\right]=L_{\left(\pi^{\prime}, \varphi_{\xi}\right)}\left[C^{\prime} ; f_{\xi}^{\prime}\right] .
$$

Note that the decomposition $C=\Sigma_{\zeta} C_{\zeta}^{\prime}$ gives the equation

$$
L_{\left(\pi^{\prime}, \varphi\right)}[C ; f]=\sum_{\zeta} L_{\left(\pi^{\prime}, \varphi\right)}\left[C_{\zeta}^{\prime} ; j_{\zeta} f i_{\zeta}\right] .
$$

But Lemma (5.4) as adjusted implies that

$$
\sum \nu_{\zeta} \zeta_{*} L_{\left(\pi^{\prime}, \varphi\right)}\left[C_{\zeta}^{\prime} ; j_{\zeta} f_{\zeta}\right] \otimes \zeta=\left|\pi_{\left[1^{\prime \prime}\right]}^{\prime \prime}\right|\left(\sum L_{\left(\pi^{\prime}, \varphi_{\xi}\right)}\left[C^{\prime} ; f_{\xi}^{\prime}\right] \otimes \xi\right)
$$

where $\pi_{\left[1^{\prime \prime}\right]}^{\prime \prime}$ is the stabilizer $\left\{\sigma^{\prime \prime} \in \pi^{\prime \prime} \mid \sigma^{\prime \prime} \varphi^{\prime \prime}\left(\sigma^{\prime \prime-1}\right)=1^{\prime \prime}\right\}$ of $1^{\prime \prime}$ in $\pi^{\prime \prime}$, and where $\zeta$ ranges over $Z$ while $\xi$ ranges over $\Xi_{1}$. Applying $\Xi_{*}^{\prime}$ to both sides of the equation and noting that the composite

$$
\mathbf{K} R_{\varphi}\left[\pi^{\prime}\right] \stackrel{\nu_{\xi} \zeta_{*}}{\rightarrow} \mathbf{K} R_{\varphi_{\xi}}\left[\pi^{\prime}\right] \stackrel{\Xi_{*}^{\prime}}{\rightarrow} \mathbf{K} R_{\varphi}[\pi]
$$

is just the natural homomorphism $\mathbf{K} R_{\varphi}\left[\pi^{\prime}\right] \rightarrow \mathbf{K} R_{\varphi}[\pi]$ which takes $\left[\gamma, \pi^{\prime}\right]_{\varphi}$ to $[\gamma, \pi]_{\varphi}$, we see that

$$
\Xi_{*}^{\prime}\left(L_{\left(\pi^{\prime}, \varphi\right)}[C ; f]\right)=\Xi_{*}^{\prime}\left(\left|\pi_{\left[1^{\prime \prime}\right]}^{\prime \prime}\right|\left(\sum_{\xi} L_{\left(\pi^{\prime}, \varphi_{\xi}\right)}\left[C^{\prime} ; f_{\xi}^{\prime}\right] \otimes \xi\right)\right) .
$$


LEMMA (5.7). Suppose that $\xi=\zeta \varphi\left(\zeta^{\prime}\right)$. Then

$$
\zeta_{*} L_{\left(\pi^{\prime}, \varphi_{\xi}\right)}\left[C ; f_{\xi}\right]=L_{\left(\pi^{\prime}, \varphi\right)}[C ; f] .
$$

Proof. Consider the commutative diagram

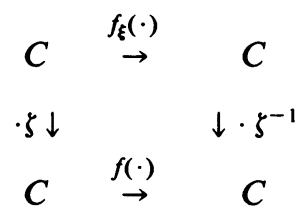

where the vertical maps are multiplications by the indicated elements. The assertion of the lemma now follows immediately by the commutativity property of the generalized Lefschetz number (Proposition (1.3)).

Again if $\xi^{\prime}=\zeta \varphi\left(\zeta^{-1}\right)$ does not lie in $\Xi$, then Lemma (5.7) can be adjusted to assert that

$$
\nu_{\zeta}^{-1} \zeta_{*}^{-1} L_{\left(\pi^{\prime}, \varphi_{\xi}\right)}\left[C ; f_{\xi}\right]=L_{\left(\pi^{\prime}, \varphi\right)}[C ; f]
$$

where $\nu_{\zeta}$ is the isomorphism

$$
\mathbf{K} R_{\varphi_{\xi}}\left[\pi^{\prime}\right] \rightarrow \mathbf{K} R_{\varphi_{\xi}}\left[\pi^{\prime}\right]
$$

induced by sending $\gamma$ to $\gamma \sigma$, where $\xi^{\prime}=\sigma \xi$, with $\sigma \in \pi^{\prime}$.

Now by Lemma (5.7) as adjusted, we have

$$
\begin{aligned}
q_{*} \sum_{\xi} L_{\left(\pi^{\prime}, \varphi_{\xi}\right)}\left[C ; f_{\xi}\right] & =\Xi_{*}^{\prime}\left(\sum_{\xi} L_{\left(\pi^{\prime}, \varphi_{\xi}\right)}\left[C ; f_{\xi}\right]\right) \\
& =\Xi_{*}^{\prime}\left(\sum_{\xi} \nu_{\xi} \zeta_{*} L_{\left(\pi^{\prime}, \varphi\right)}[C ; f]\right) \\
& =\left(q_{*} L_{\left(\pi^{\prime}, \varphi\right)}[C ; f]\right) \cdot\left|\pi^{\prime \prime} / \pi_{\left[1^{\prime \prime}\right]}^{\prime}\right|
\end{aligned}
$$

where $\xi$ ranges over the elements of $\Xi$. But, since $q_{*}=\Xi_{*}^{\prime}$ on the submodule $\mathrm{K} R_{\varphi}\left[\pi^{\prime}\right]$ of $\mathrm{K} R_{\varphi}\left[\pi, \pi^{\prime}\right]$, equations (5.5) and (5.6) imply that

$$
q_{*} L_{\left(\pi^{\prime}, \varphi\right)}[C ; f]=\left|\pi_{\left[1^{\prime \prime}\right]}^{\prime \prime}\right| \Xi_{*}^{\prime}\left(\sum L_{\left(\pi^{\prime}, \varphi_{\xi}\right)}\left[C^{\prime} ; f_{\xi}^{\prime}\right] \otimes \xi\right) .
$$

Thus we obtain the equation

$$
q_{*}\left(\sum_{\xi} L_{\left(\pi^{\prime}, \varphi\right)}\left[C ; f_{\xi}\right]\right)=\left|\pi^{\prime \prime}\right| \Xi_{*}^{\prime}\left(\sum L_{\left(\pi^{\prime}, \varphi_{\xi}\right)}\left[C^{\prime} ; f_{\xi}^{\prime}\right] \otimes \xi\right)
$$

where $\xi$ ranges over $\Xi_{1}$. Next we note that Theorem (1.7) implies that

$$
\begin{aligned}
\left(q_{*} L_{\left(\pi^{\prime}, \varphi\right)}\left[C, C^{\prime} ; f\right]\right)_{1} & =\Xi_{*} \Xi_{*}^{\prime}\left(L_{\left(\pi^{\prime}, \varphi\right)}\left[C, C^{\prime} ; f\right]\right) \\
& =\Xi_{*}\left(\sum_{\xi} L_{\left(\pi^{\prime}, \varphi_{\xi}\right)}\left[C^{\prime} ; f_{\xi}^{\prime}\right] \otimes \xi\right)
\end{aligned}
$$


where $L_{\left(\pi^{\prime}, \varphi\right)}\left[C, C^{\prime} ; f\right]_{\omega}$ denotes the terms which involve those elements in $\amalg_{\xi \in \Xi_{\omega}} R_{\varphi}\left[\pi_{\xi}^{\prime}, \pi^{\prime}\right]$. But

$$
q_{*} L_{\left(\pi^{\prime}, \varphi\right)}\left[C, C^{\prime} ; f\right]_{1}=L_{(\pi, \varphi)}[C ; f]_{1}
$$

which proves that equation (5.3) 1 holds.

The proof of $(5.3)_{\omega}$ when $\omega \neq 1$ proceeds as follows. First observe that the correspondence $\gamma \rightarrow \gamma \omega^{-1}$ induces an isomorphism

$$
\mathbf{K} R_{\varphi}[\pi] \rightarrow \mathbf{K} R_{\varphi_{\omega}}[\pi]
$$

which takes $L_{(\pi, \varphi)}[C ; f]_{\omega}$ to $L_{\left(\pi, \varphi_{\omega}\right)}\left[C ; f_{\omega}\right]$. Now with $\Xi$ replaced by $H=\Xi_{\omega}, f$ by $g=f_{\omega}$ and $\varphi$ by $\gamma=\varphi_{\omega}$, we can conclude that

$$
\left|\pi^{\prime \prime}\right| L_{(\pi, \psi)}[C ; g]=H_{*}^{\prime}\left(\sum_{\eta} L_{\left(\pi^{\prime}, \psi_{\eta}\right)}[C ; g] \otimes \eta\right)
$$

as $\eta$ ranges over $H_{1}=\left\{\eta \in H \mid \eta=\rho \psi\left(\rho^{-1}\right) \bmod \pi^{\prime}, \rho \in \pi\right\}$. Also the correspondence $\gamma \mapsto \gamma \omega^{-1}$ induces an isomorphism $\mathbf{K} R_{\varphi}\left[\pi, \pi^{\prime}\right] \rightarrow \mathbf{K} R_{\psi}\left[\pi, \pi^{\prime}\right]$ which takes $\left(L_{\left(\pi^{\prime}, \varphi\right)}\left[C, C^{\prime} ; f\right]\right)_{\omega}$ to $\left(L_{\left(\pi^{\prime}, \psi\right)}\left[C, C^{\prime} ; g\right]\right)_{1}$. Moreover the correspondence $\left(\gamma^{\prime}, \xi\right) \mapsto\left(\gamma^{\prime}, \xi \omega^{-1}\right)$ induces an isomorphism

$$
\sum_{\xi \in \Xi} \mathrm{K} R_{\varphi_{\xi}}\left[\pi^{\prime}\right] \otimes \xi \rightarrow \sum_{\eta \in H} \mathrm{~K} R_{\psi_{\eta}}\left[\pi^{\prime}\right] \otimes \eta
$$

which takes

$$
\sum_{\xi \in \Xi_{\omega}} L_{\left(\pi^{\prime}, \varphi_{\xi}\right)}\left[C ; f_{\xi}\right] \otimes \xi
$$

to

$$
\sum_{\eta \in H_{1}} L_{\left(\pi^{\prime}, \psi_{\eta}\right)}\left[C ; g_{\eta}\right] \otimes \eta
$$

Now it is easy to deduce $(5.3)_{\omega}$, namely that

$$
\left(\left|\pi^{\prime \prime}\right| L_{(\pi, \varphi)}[C ; f]\right)_{\omega}=\Xi_{*}^{\prime}\left(\sum_{\xi \in \Xi_{\omega}} L_{\left(\pi^{\prime}, \varphi_{\xi}\right)}\left[C, f_{\xi}\right]\right),
$$

and this finishes the proof of the theorem.

6. Proofs of Propositions (1.12) and (1.13) and Theorem (1.14). The proof of Proposition (1.9) depends on the following proposition. Suppose that

$$
\begin{array}{cccc} 
& X^{\prime \prime} & \stackrel{f^{\prime \prime}}{\rightarrow} & X^{\prime \prime} \\
S_{\left(\pi^{\prime \prime}, \varphi^{\prime \prime}\right)}[X, f]: & \downarrow & & \downarrow \\
& X & \stackrel{f}{\rightarrow} & X
\end{array}
$$

is a $\left(\pi^{\prime \prime}, \varphi^{\prime \prime}\right)$-setting, where $X$ is a CW-complex, and let

$$
F: X \times I \rightarrow X \times I
$$


be a homotopy, written as a level-preserving map, such that $F \mid X \times\{0\}=f$. Then, by the covering homotopy property, we obtain a unique $\left(\pi^{\prime \prime}, \varphi^{\prime \prime}\right)$-setting

$$
\begin{array}{cccc} 
& X^{\prime \prime} \times I & \stackrel{F^{\prime \prime}}{\rightarrow} & X^{\prime \prime} \times I \\
S_{\left(\pi^{\prime \prime}, \varphi^{\prime \prime}\right)}[X \times I, F] & \downarrow & & \downarrow \\
& X \times I & \stackrel{F}{\rightarrow} & X \times I
\end{array}
$$

such that

$$
S_{\left(\pi^{\prime \prime}, \varphi^{\prime \prime}\right)}[X \times\{0\}, F \mid X \times\{0\}]=S_{\left(\pi^{\prime \prime}, \varphi^{\prime \prime}\right)}[X, f] .
$$

Proposition (6.1). Suppose that $f$ and $g: X \rightarrow X$, where $g=F \mid X \times\{1\}$, are both cellular. Then

$$
L_{\left(\pi^{\prime \prime}, \varphi^{\prime \prime}\right)}\left[C_{*} \tilde{X}^{\prime \prime} ; C_{*} \tilde{f}^{\prime \prime}\right]=L_{\left(\pi^{\prime \prime}, \varphi^{\prime \prime}\right)}\left[C_{*} \tilde{X}^{\prime \prime}, C_{*} \tilde{g}^{\prime \prime}\right]
$$

where $C_{*} \tilde{X}^{\prime \prime}$ are the cellular chains of $\tilde{X}^{\prime \prime}$, and $\tilde{f}^{\prime \prime}$ and $\tilde{g}^{\prime \prime}$ are the lifts of $f$ and $g$ provided by the setting $S_{\left(\pi^{\prime \prime}, \varphi^{\prime \prime}\right)}[X \times I, F]$.

Proof. We can assume without loss of generality that $F$ is cellular, for we can replace $F: X \times I \rightarrow X \times I$ by a cellular approximation without altering it at either end, and then deform the setting $S_{\left(\pi^{\prime \prime}, \varphi^{\prime \prime}\right)}[X \times I, F]$ accordingly. But if $F$ is cellular then the proposition follows immediately from Proposition (3.2).

Now suppose that $S_{\left(\pi^{\prime \prime}, \varphi^{\prime \prime}\right)}[X, f]$ is any setting with $X$ being a finite $\mathrm{CW}$-complex. Choose any cellular approximation $f^{c}: X \rightarrow X$ for $f$ and alter the $\left(\pi^{\prime \prime}, \varphi^{\prime \prime}\right)$-setting accordingly. As in $§ 1$, by definition, let

$$
\left.L_{\left(\pi^{\prime \prime}, \varphi^{\prime \prime}\right)} S[X, f]=L_{\left(\pi^{\prime \prime}, \varphi^{\prime \prime}\right)}\left[C_{*} X^{\prime \prime} ; C_{*} f^{c}\right)^{\prime \prime}\right] \text {. }
$$

Since any two cellular approximations of $f$ are homotopic, we see as a consequence of Proposition (6.1) that $L_{\left(\pi^{\prime \prime}, \varphi^{\prime \prime}\right)} S[X, f]$ is independent of the cellular approximation $f^{c}: X \rightarrow X$, and hence it is well defined.

The homotopy invariance of the generalized Lefschetz number (Proposition (1.11)) can be deduced from Proposition (6.1) in a similar fashion: if $f, g: X \rightarrow X$ are homotopic, then any cellular approximation of $f$ is homotopic to any cellular approximation of $g$, and thus Proposition (1.11) follows immediately from Proposition (6.1).

If $X$ is a simplicial complex then the generalized Lefschetz numbers $L_{\left(\pi^{\prime \prime}, \varphi^{\prime \prime}\right)}$ can be computed by means of the simplicial structure as follows. Suppose that $f^{s}: X^{s} \rightarrow X$ is a simplicial approximation of $f$, with $X^{s}$ being a simplicial subdivision of $X$. As $f^{s}$ is homotopic to $f$, the given $\left(\pi^{\prime \prime}, \varphi^{\prime \prime}\right)$-setting $S_{\left(\pi^{\prime \prime}, \varphi^{\prime \prime}\right)}[X, f]$ induces a commutative diagram

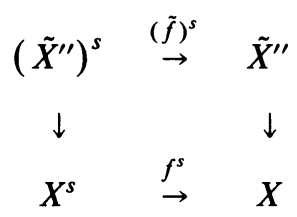


where $\left(\tilde{X}^{\prime \prime}\right)^{s}$ is the subdivision of $\tilde{X}^{\prime \prime}$ induced by the subdivision $X^{s}$ of $X$, and $\left(\tilde{f}^{\prime \prime}\right)^{s}$ is the $\left(\pi^{\prime \prime}, \varphi^{\prime \prime}\right)$-simplicial map over $f^{s}$. Denote by

$$
C_{*}\left(\tilde{f}^{\prime \prime}\right)^{s}: C_{*}\left(\tilde{X}^{\prime \prime}\right)^{s} \rightarrow C_{*}\left(\tilde{X}^{\prime \prime}\right)^{s}
$$

the composite

$$
C_{*}\left(\tilde{X}^{\prime \prime}\right)^{s} \stackrel{C_{*}\left(f^{\prime \prime}\right)^{s}}{\rightarrow} C_{*} \tilde{X}^{\prime \prime} \stackrel{\text { Sd }}{\rightarrow} C_{*}\left(X^{\prime \prime}\right)^{s}
$$

where $\mathrm{Sd}$ is the $\pi^{\prime \prime}$-chain map induced by barycentric subdivision.

PROPOSITION (6.2). With the notation as above, we have

$$
L_{\left(\pi^{\prime \prime}, \varphi^{\prime \prime}\right)} S[X, f]=L_{\left(\pi^{\prime \prime}, \varphi^{\prime \prime}\right)}\left[C_{*}\left(\tilde{X}^{\prime \prime}\right)^{s} ; C_{*}\left(\tilde{f}^{\prime \prime}\right)^{s}\right] .
$$

Proof. Let $h: X \rightarrow X^{s}$ be a cellular approximation of the identity map

$$
X \stackrel{=}{\rightarrow} X^{s},
$$

where we regard $X$ and $X^{s}$ as cellular complexes. Note that the cellular chain groups of $\tilde{X}^{\prime \prime}$ and $\left(\tilde{X}^{\prime \prime}\right)^{s}$ regarded as $\mathrm{CW}$-complexes are the same as their simplicial groups [2] and consider the following diagram

$$
\begin{array}{ccccc}
C_{*} \tilde{X}^{\prime \prime} & \stackrel{C_{*}\left(\tilde{g}^{\prime \prime}\right)}{\rightarrow} & C_{*} \tilde{X}^{\prime \prime} & \stackrel{=}{\rightarrow} & C_{*} X^{\prime \prime} \\
C_{*}(\tilde{h})^{\prime \prime} \downarrow & & \downarrow= & & \downarrow C_{*}(\tilde{h})^{\prime \prime} \\
& & \downarrow & & \\
C_{*}^{*}\left(\tilde{X}^{\prime \prime}\right)^{s} & \stackrel{C_{*}\left(\tilde{f}^{\prime \prime}\right)^{s}}{\rightarrow} & C_{*} \tilde{X}^{\prime \prime} & \stackrel{\text { Sd }}{\rightarrow} & \left(C_{*} \tilde{X}^{\prime \prime}\right)^{s}
\end{array}
$$

where $\tilde{h}$ is a lift of $h, \tilde{g}^{\prime \prime}=(\tilde{f})^{s} \circ \tilde{h}^{\prime \prime}$ and Sd the chain map induced by barycentric subdivision. The square on the left is commutative by definition while the square on the right commutes up to homotopy, since $\mathrm{Sd}$ is $\pi^{\prime \prime}$-chain homotopy equivalent to any of the $\pi^{\prime \prime}$-chain maps induced by the projections $\left(\tilde{X}^{\prime \prime}\right)^{s} \rightarrow \tilde{X}^{\prime \prime}$. (The projections are simplicial approximations of the identity map $\left(\tilde{X}^{\prime \prime}\right)^{s} \rightarrow \tilde{X}^{\prime \prime}$.) Now the proposition follows immediately from Proposition (3.2).

Proof of Proposition (1.12). Replace $f: X \rightarrow Y$ and $g: Y \rightarrow X$ by cellular approximations $f^{c}$ and $g^{c}$ and note that $g^{c} f^{c}$ and $f^{c} g^{c}$ are cellular approximations of $g f$ and $f g$ respectively, and hence can be used to compute the generalized Lefschetz numbers. But then Proposition (1.12) is just an immediate consequence of Proposition (1.2).

Proof OF Theorem (1.13). Suppose that

$$
\begin{array}{cccc} 
& \tilde{X}^{\prime \prime} & \stackrel{\tilde{f}^{\prime \prime}}{\rightarrow} & \tilde{X}^{\prime \prime} \\
S_{\left(\pi^{\prime \prime}, \varphi^{\prime \prime}\right)}[X, f]: & \downarrow & & \downarrow \\
& X & \stackrel{f}{\rightarrow} & X
\end{array}
$$


is a $\left(\pi^{\prime \prime}, \varphi^{\prime \prime}\right)$-setting with $X$ being a finite CW-complex. Imbed $X$ as a retract $X \subset^{i} Y$ in a polyhedron $Y$ and denote the retraction $Y \rightarrow X$ by $j$. Now the pull-back of $S_{\left(\pi^{\prime \prime}, \varphi^{\prime \prime}\right)}[X, f]$ by $j$ is a $\left(\pi^{\prime \prime}, \varphi^{\prime \prime}\right)$-setting

$$
\begin{array}{cccc} 
& Y^{\prime \prime} & \stackrel{(\tilde{f})^{\prime \prime}(\tilde{j})^{\prime \prime}}{\rightarrow} & Y^{\prime \prime} \\
S_{\left(\pi^{\prime \prime}, \varphi^{\prime \prime}\right)}[Y, i f j] & \downarrow & & \downarrow \\
& Y & \stackrel{(i f) j}{\rightarrow} & Y
\end{array}
$$

where $(\widetilde{i f})^{\prime \prime}$ and $\tilde{j^{\prime \prime}}$ are lifts of if and $j$ which fit in the commutative diagram:

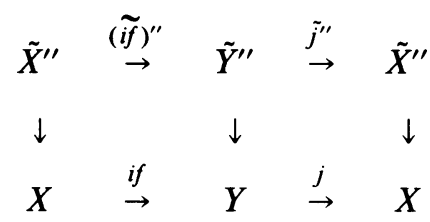

Now according to Proposition (1.12) we have the equality

$$
L_{\left(\pi^{\prime \prime}, \varphi^{\prime \prime}\right)} S[X, f]=L_{\left(\pi^{\prime \prime}, \varphi^{\prime \prime}\right)} S[Y, \text { ifj }] .
$$

Note that

$$
\operatorname{Fix}(f)=\operatorname{Fix}(i f j)
$$

and that the $\pi^{\prime \prime}$-Nielsen classes of $\operatorname{Fix}(f)$ agree with those of $\operatorname{Fix}(i f j)$. Thus it suffices to prove Theorem (1.13) for $g=i f j: Y \rightarrow Y$. The rest of the argument is essentially Wecken's computation [8]. Replace $g$ by a simplicial approximation $g^{s}$ : $Y^{s} \rightarrow Y$ of the Hopf type. That is to say $g^{s}$ fixes only maximal simplices and each fixed simplex contains only one fixed point. Using the $\left(\pi^{\prime \prime}, \varphi^{\prime \prime}\right)$-chain complex $\left[C\left(\tilde{Y}^{\prime \prime}\right)^{s}, C_{*}\left(\tilde{g}^{\prime \prime}\right)^{s}\right]$ one proceeds to compute the trace directly. It is clear that we need only consider the chains in the top dimension. Note that only those simplices which are fixed by $g^{s}$ contribute to the trace, and if $\{\tilde{x}\}$ is a basis for $C_{*}\left(\tilde{Y}^{\prime \prime}\right)^{s}$, where each $\tilde{x}$ is a lift of a simplex $x$ of $Y$ maximal dimension, and if $x$ is a simplex fixed by $g^{s}$, then the contribution of $\tilde{x}$ to the trace is exactly $\eta_{x} \alpha_{x}$ where the integer $\eta_{x}$ is the index, in the usual sense, of the fixed point contained in $x$ and $\alpha_{x}$ is that element in $\pi^{\prime \prime}$ with the property that $\left(\tilde{g}^{\prime \prime}\right)^{s}(x) \alpha^{-1}=\left(g^{\prime \prime}\right)_{\alpha}^{s}(x) \supset x$. Finally one verifies easily that $\left[\alpha_{x} ; \pi^{\prime \prime}\right]_{\varphi^{\prime \prime}}=\left[\alpha_{x^{\prime}} ; \pi^{\prime \prime}\right]_{\varphi^{\prime \prime}}$ if and only if the lifts $\left(\tilde{g}^{\prime \prime}\right)_{\alpha_{x}}^{s}$ and $\left(\tilde{g}^{\prime \prime}\right)_{\alpha_{x^{\prime}}}^{s}$ are conjugate by an element in $\pi^{\prime \prime}$. Hence $\alpha_{x}$ and $\alpha_{x^{\prime}}$ are $\left(\pi^{\prime \prime}, \varphi^{\prime \prime}\right)$-Reidemeister equivalent if and only if $x$ and $x^{\prime}$ are $\pi^{\prime \prime}$-Nielsen equivalent, and since $\Sigma \eta_{x}$, as $x$ ranges over the points of the $\pi^{\prime \prime}$-Nielsen class $\{x\}$, is exactly the index of $\{x\}$, we see that $\lambda_{\left[\alpha_{x} ; \pi^{\prime \prime}\right]_{\varphi^{\prime \prime}}}$ is equal to the index of $\{x\}$ as required.

\section{REFERENCES}

1. R. F. Brown, The Lefschetz Fixed Point Theorem, Scott, Foresman \& Co., Glenview, Ill., 1971.

2. S. Eilenberg and N. E. Steenrod, Foundations of algebraic topology, Princeton Univ. Press, Princeton, N. J., 1951.

3. E. Fadell and S. Y. Husseini, Fixed point theory for non-simply connected manifolds, Topology 20 (1981), 53-92. 
4. D. H. Gottlieb, A certain subgroup of the fundamental group, Amer. J. Math. 87 (1965), 840-856.

5. D. McCord, An estimate of the Nielsen number and an example concerning the Lefschetz Fixed Point Theorem, Pacific J. Math. 66 (1976), 195-203.

6. J. Stallings, Centerless groups - an algebraic formulation of Gottlieb's Theorem, Topology 4 (1965), 129-134.

7. K. Reidemeister, Automorphismen von Homotopie Kettenringen, Math. Ann. 112 (1936), 586-593.

8. F. Wecken, Fixpunktklassen. II, Math. Ann. 118 (1941/43), 216-234.

Department of Mathematics, University of Wisconsin, Madison, Wisconsin 53706 